DESY 98-204

ISSN 0418-9833

December 1998

\title{
Measurement of $D^{*}$ Meson Cross Sections at HERA and Determination of the Gluon Density in the Proton using NLO QCD
}

\author{
H1 Collaboration
}

\begin{abstract}
With the $\mathrm{H} 1$ detector at the ep collider HERA, $D^{*}$ meson production cross sections have been measured in deep inelastic scattering with four-momentum transfers $Q^{2}>2 \mathrm{GeV}^{2}$ and in photoproduction at energies around $W_{\gamma p} \approx 88 \mathrm{GeV}$ and $194 \mathrm{GeV}$. Next-to-Leading Order QCD calculations are found to describe the differential cross sections within theoretical and experimental uncertainties. Using these calculations, the NLO gluon momentum distribution in the proton, $x_{g} g\left(x_{g}\right)$, has been extracted in the momentum fraction range $7.5 \cdot 10^{-4}<x_{g}<4 \cdot 10^{-2}$ at average scales $\mu^{2}=25$ to $50 \mathrm{GeV}^{2}$. The gluon momentum fraction $x_{g}$ has been obtained from the measured kinematics of the scattered electron and the $D^{*}$ meson in the final state. The results compare well with the gluon distribution obtained from the analysis of scaling violations of the proton structure function $F_{2}$.
\end{abstract}

Submitted to Nuclear Physics B 
C. Adloff ${ }^{34}$, M. Anderson ${ }^{22}$, V. Andreev ${ }^{25}$, B. Andrieu ${ }^{28}$, V. Arkadov ${ }^{35}$, C. Arndt ${ }^{11}$, I. Ayyaz ${ }^{29}$, A. Babaev ${ }^{24}$, J. Bähr ${ }^{35}$, P. Baranov ${ }^{25}$, E. Barrelet ${ }^{29}$, W. Bartel ${ }^{11}$, U. Bassler ${ }^{29}$, P. Bate ${ }^{22}$, M. Beck ${ }^{13}$, A. Beglarian ${ }^{11,40}$, O. Behnke ${ }^{11}$, H.-J. Behrend ${ }^{11}$, C. Beier ${ }^{15}$, A. Belousov ${ }^{25}$, Ch. Berger ${ }^{1}$, G. Bernardi ${ }^{29}$, G. Bertrand-Coremans ${ }^{4}$, P. Biddulph ${ }^{22}$, J.C. Bizot ${ }^{27}$, V. Boudry ${ }^{28}$, W. Braunschweig ${ }^{1}$, V. Brisson ${ }^{27}$, D.P. Brown ${ }^{22}$, W. Brückner ${ }^{13}$, P. Bruel ${ }^{28}$, D. Bruncko ${ }^{17}$, J. Bürger ${ }^{11}$, F.W. Büsser ${ }^{12}$, A. Buniatian ${ }^{32}$, S. Burke ${ }^{18}$, A. Burrage ${ }^{19}$, G. Buschhorn ${ }^{26}$, D. Calvet ${ }^{23}$, A.J. Campbell ${ }^{11}$, T. Carli ${ }^{26}$, E. Chabert ${ }^{23}$, M. Charlet ${ }^{4}$, D. Clarke ${ }^{5}$, B. Clerbaux ${ }^{4}$, J.G. Contreras ${ }^{8,42}$, C. Cormack ${ }^{19}$, J.A. Coughlan ${ }^{5}$, M.-C. Cousinou ${ }^{23}$, B.E. Cox $^{22}$, G. Cozzika ${ }^{10}$, J. Cvach ${ }^{30}$, J.B. Dainton ${ }^{19}$, W.D. Dau ${ }^{16}$, K. Daum ${ }^{39}$, M. David ${ }^{10}$, M. Davidsson ${ }^{21}$, A. De Roeck ${ }^{11}$, E.A. De Wolf ${ }^{4}$, B. Delcourt ${ }^{27}$, R. Demirchyan ${ }^{11,40}$, C. Diaconu ${ }^{23}$, M. Dirkmann ${ }^{8}$, P. Dixon ${ }^{20}$, W. Dlugosz ${ }^{7}$, K.T. Donovan ${ }^{20}$, J.D. Dowell ${ }^{3}$, A. Droutskoi ${ }^{24}$, J. Ebert $^{34}$, G. Eckerlin ${ }^{11}$, D. Eckstein ${ }^{35}$, V. Efremenko ${ }^{24}$, S. Egli $^{37}$, R. Eichler ${ }^{36}$, F. Eisele ${ }^{14}$, E. Eisenhandler ${ }^{20}$, E. Elsen ${ }^{11}$, M. Enzenberger ${ }^{26}$, M. Erdmann $^{14,43, f}$, A.B. Fahr ${ }^{12}$, L. Favart ${ }^{4}$, A. Fedotov ${ }^{24}$, R. Felst ${ }^{11}$, J. Feltesse ${ }^{10}$, J. Ferencei ${ }^{17}$, F. Ferrarotto ${ }^{32}$, M. Fleischer ${ }^{8}$, G. Flügge ${ }^{2}$, A. Fomenko ${ }^{25}$, J. Formánek ${ }^{31}$, J.M. Foster ${ }^{22}$, G. Franke ${ }^{11}$, E. Gabathuler ${ }^{19}$, K. Gabathuler ${ }^{33}$, F. Gaede ${ }^{26}$, J. Garvey ${ }^{3}$, J. Gassner ${ }^{33}$, J. Gayler ${ }^{11}$, R. Gerhards ${ }^{11}$, S. Ghazaryan ${ }^{11,40}$, A. Glazov ${ }^{35}$, L. Goerlich ${ }^{6}$, N. Gogitidze ${ }^{25}$, M. Goldberg ${ }^{29}$, I. Gorelov ${ }^{24}$, C. Grab ${ }^{36}$, H. Grässler ${ }^{2}$, T. Greenshaw ${ }^{19}$, R.K. Griffiths ${ }^{20}$, G. Grindhammer ${ }^{26}$, T. Hadig ${ }^{1}$, D. Haidt ${ }^{11}$, L. Hajduk ${ }^{6}$, T. Haller ${ }^{13}$, M. Hampel ${ }^{1}$, V. Haustein ${ }^{34}$, W.J. Haynes ${ }^{5}$, B. Heinemann ${ }^{11}$, G. Heinzelmann ${ }^{12}$, R.C.W. Henderson ${ }^{18}$, S. Hengstmann ${ }^{37}$, H. Henschel ${ }^{35}$, R. Heremans ${ }^{4}$, I. Herynek ${ }^{30}$, K. Hewitt ${ }^{3}$, K.H. Hiller ${ }^{35}$, C.D. Hilton ${ }^{22}$, J. Hladký ${ }^{30}$, D. Hoffmann ${ }^{11}$, R. Horisberger ${ }^{33}$, S. Hurling ${ }^{11}$, M. Ibbotson ${ }^{22}$, Ç. İssever ${ }^{8}$, M. Jacquet $^{27}$, M. Jaffre ${ }^{27}$, D.M. Jansen ${ }^{13}$, L. Jönsson ${ }^{21}$, D.P. Johnson ${ }^{4}$, M. Jones ${ }^{19}$, H. Jung ${ }^{21}$, H.K. Kästli ${ }^{36}$, M. Kander ${ }^{11}$, D. Kant ${ }^{20}$, M. Kapichine ${ }^{9}$, M. Karlsson ${ }^{21}$, O. Karschnik ${ }^{12}$, J. Katzy ${ }^{11}$, O. Kaufmann ${ }^{14}$, M. Kausch ${ }^{11}$, I.R. Kenyon ${ }^{3}$, S. Kermiche ${ }^{23}$, C. Keuker ${ }^{1}$, C. Kiesling ${ }^{26}$, M. Klein ${ }^{35}$, C. Kleinwort ${ }^{11}$, G. Knies ${ }^{11}$, J.H. Köhne ${ }^{26}$, H. Kolanoski ${ }^{38}$, S.D. Kolya ${ }^{22}$, V. Korbel ${ }^{11}$, P. Kostka ${ }^{35}$, S.K. Kotelnikov ${ }^{25}$, T. Krämerkämper ${ }^{8}$, M.W. Krasny ${ }^{29}$, H. Krehbiel ${ }^{11}$, D. $\operatorname{Krücker~}^{26}$, K. Krüger ${ }^{11}$, A. Küpper ${ }^{34}$, H. Küster ${ }^{2}$, M. Kuhlen ${ }^{26}$, T. Kurča ${ }^{35}$, R. Lahmann ${ }^{11}$, M.P.J. Landon ${ }^{20}$, W. Lange ${ }^{35}$, U. Langenegger ${ }^{36}$, A. Lebedev ${ }^{25}$, F. Lehner ${ }^{11}$, V. Lemaitre ${ }^{11}$, V. Lendermann ${ }^{8}$, S. Levonian ${ }^{11}$, M. Lindstroem ${ }^{21}$, B. List ${ }^{11}$, G. Lobo ${ }^{27}$, E. Lobodzinska ${ }^{6,41}$, V. Lubimov ${ }^{24}$, S. Lüders ${ }^{36}$, D. Lüke ${ }^{8,11}$, L. Lytkin ${ }^{13}$, N. Magnussen ${ }^{34}$, H. Mahlke-Krüger ${ }^{11}$, E. Malinovski ${ }^{25}$, R. Maraček ${ }^{17}$, P. Marage ${ }^{4}$, J. Marks $^{14}$, R. Marshall ${ }^{22}$, G. Martin ${ }^{12}$, H.-U. Martyn ${ }^{1}$, J. Martyniak ${ }^{6}$, S.J. Maxfield ${ }^{19}$, T.R. McMahon ${ }^{19}$, A. Mehta ${ }^{5}$, K. Meier ${ }^{15}$, P. Merkel ${ }^{11}$, F. Metlica ${ }^{13}$, A. Meyer ${ }^{11}$, A. Meyer ${ }^{11}$, H. Meyer ${ }^{34}$, J. Meyer ${ }^{11}$, P.-O. Meyer ${ }^{2}$, S. Mikocki ${ }^{6}$, D. Milstead ${ }^{11}$, J. Moeck ${ }^{26}$, R. Mohr ${ }^{26}$, S. Mohrdieck ${ }^{12}$, F. Moreau ${ }^{28}$, J.V. Morris ${ }^{5}$, D. Müller ${ }^{37}$, K. Müller ${ }^{11}$, P. Murin ${ }^{17}$, V. Nagovizin ${ }^{24}$, B. Naroska ${ }^{12}$, Th. Naumann ${ }^{35}$, I. Négri ${ }^{23}$, P.R. Newman ${ }^{3}$, H.K. Nguyen ${ }^{29}$, T.C. Nicholls ${ }^{11}$, F. Niebergall ${ }^{12}$, C. Niebuhr ${ }^{11}$, Ch. Niedzballa ${ }^{1}$, H. Niggli ${ }^{36}$, D. Nikitin ${ }^{9}$, O. Nix ${ }^{15}$, G. Nowak ${ }^{6}$, T. Nunnemann ${ }^{13}$, H. Oberlack ${ }^{26}$, J.E. Olsson ${ }^{11}$, D. Ozerov ${ }^{24}$, P. Palmen ${ }^{2}$, V. Panassik ${ }^{9}$, C. Pascaud ${ }^{27}$, S. Passaggio ${ }^{36}$, G.D. Patel ${ }^{19}$, H. Pawletta ${ }^{2}$, E. Perez ${ }^{10}$, J.P. Phillips ${ }^{19}$, A. Pieuchot ${ }^{11}$, D. Pitzl ${ }^{36}$, R. Pöschl ${ }^{8}$, G. Pope ${ }^{7}$, B. Povh ${ }^{13}$, K. Rabbertz ${ }^{1}$, J. Rauschenberger ${ }^{12}$, P. Reimer ${ }^{30}$, B. Reisert ${ }^{26}$, D. Reyna ${ }^{11}$, H. Rick ${ }^{11}$, S. Riess ${ }^{12}$, E. Rizvi ${ }^{19}$, P. Robmann ${ }^{37}$, R. Roosen ${ }^{4}$, K. Rosenbauer ${ }^{1}$, A. Rostovtsev ${ }^{24,12}$, F. Rouse ${ }^{7}$, C. Royon $^{10}$, S. Rusakov ${ }^{25}$, K. Rybicki ${ }^{6}$, D.P.C. Sankey ${ }^{5}$, P. Schacht ${ }^{26}$, J. Scheins ${ }^{1}$, F.-P. Schilling ${ }^{14}$, S. Schleif ${ }^{15}$, P. Schleper ${ }^{14}$, D. Schmidt ${ }^{34}$, D. Schmidt ${ }^{11}$, L. Schoeffel ${ }^{10}$, V. Schröder ${ }^{11}$, H.-C. Schultz-Coulon ${ }^{11}$, B. Schwab ${ }^{14}$, F. Sefkow ${ }^{37}$, A. Semenov ${ }^{24}$, V. Shekelyan ${ }^{26}$, I. Sheviakov ${ }^{25}$, L.N. Shtarkov ${ }^{25}$, G. Siegmon ${ }^{16}$, 
Y. Sirois ${ }^{28}$, T. Sloan ${ }^{18}$, P. Smirnov ${ }^{25}$, M. Smith ${ }^{19}$, V. Solochenko ${ }^{24}$, Y. Soloviev ${ }^{25}$, V. Spaskov ${ }^{9}$, A. Specka ${ }^{28}$, J. Spiekermann ${ }^{8}$, H. Spitzer ${ }^{12}$, F. Squinabol ${ }^{27}$, P. Steffen ${ }^{11}$, R. Steinberg ${ }^{2}$, J. Steinhart ${ }^{12}$, B. Stella ${ }^{32}$, A. Stellberger ${ }^{15}$, J. Stiewe ${ }^{15}$, U. Straumann ${ }^{14}$, W. Struczinski ${ }^{2}$, J.P. Sutton ${ }^{3}$, M. Swart ${ }^{15}$, S. Tapprogge ${ }^{15}$, M. Taševský ${ }^{30}$, V. Tchernyshov ${ }^{24}$,

S. Tchetchelnitski ${ }^{24}$, J. Theissen $^{2}$, G. Thompson ${ }^{20}$, P.D. Thompson ${ }^{3}$, N. Tobien ${ }^{11}$, R. Todenhagen ${ }^{13}$, P. Truöl ${ }^{37}$, G. Tsipolitis ${ }^{36}$, J. Turnau ${ }^{6}$, E. Tzamariudaki ${ }^{26}$, S. Udluft $^{26}$, A. Usik ${ }^{25}$, S. Valkár ${ }^{31}$, A. Valkárová ${ }^{31}$, C. Vallée ${ }^{23}$, P. Van $\operatorname{Esch}^{4}$, A. Van Haecke ${ }^{10}$, P. Van Mechelen ${ }^{4}$, Y. Vazdik ${ }^{25}$, G. Villet ${ }^{10}$, K. Wacker ${ }^{8}$, R. Wallny ${ }^{14}$, T. Walter $^{37}$, B. Waugh ${ }^{22}$, G. Weber ${ }^{12}$, M. Weber ${ }^{15}$, D. Wegener ${ }^{8}$, A. Wegner ${ }^{26}$, T. Wengler ${ }^{14}$, M. Werner ${ }^{14}$, L.R. West ${ }^{3}$, S. Wiesand ${ }^{34}$, T. Wilksen ${ }^{11}$, S. Willard ${ }^{7}$, M. Winde ${ }^{35}$, G.-G. Winter ${ }^{11}$, C. Wittek $^{12}$, E. Wittmann ${ }^{13}$, M. Wobisch ${ }^{2}$, H. Wollatz ${ }^{11}$, E. Wünsch ${ }^{11}$, J. Žáček ${ }^{31}$, J. Zálešák ${ }^{31}$, Z. Zhang ${ }^{27}$, A. Zhokin ${ }^{24}$, P. Zini ${ }^{29}$, F. Zomer ${ }^{27}$, J. Zsembery ${ }^{10}$ and M. zurNedden ${ }^{37}$

${ }^{1}$ I. Physikalisches Institut der RWTH, Aachen, Germany ${ }^{a}$

${ }^{2}$ III. Physikalisches Institut der RWTH, Aachen, Germany ${ }^{a}$

${ }^{3}$ School of Physics and Space Research, University of Birmingham, Birmingham, $U K^{b}$

${ }^{4}$ Inter-University Institute for High Energies ULB-VUB, Brussels; Universitaire Instelling Antwerpen, Wilrijk; Belgium ${ }^{c}$

${ }^{5}$ Rutherford Appleton Laboratory, Chilton, Didcot, $U K^{b}$

${ }^{6}$ Institute for Nuclear Physics, Cracow, Poland ${ }$

${ }^{7}$ Physics Department and IIRPA, University of California, Davis, California, USA ${ }^{e}$

${ }^{8}$ Institut für Physik, Universität Dortmund, Dortmund, Germany ${ }^{a}$

${ }^{9}$ Joint Institute for Nuclear Research, Dubna, Russia

${ }^{10}$ DSM/DAPNIA, CEA/Saclay, Gif-sur-Yvette, France

${ }^{11}$ DESY, Hamburg, Germany ${ }^{a}$

12 II. Institut für Experimentalphysik, Universität Hamburg, Hamburg, Germany ${ }^{a}$

${ }^{13}$ Max-Planck-Institut für Kernphysik, Heidelberg, Germany ${ }^{a}$

${ }^{14}$ Physikalisches Institut, Universität Heidelberg, Heidelberg, Germany ${ }^{a}$

${ }^{15}$ Institut für Hochenergiephysik, Universität Heidelberg, Heidelberg, Germany ${ }^{a}$

${ }^{16}$ Institut für experimentelle und angewandte Physik, Universität Kiel, Kiel, Germany ${ }^{a}$

${ }^{17}$ Institute of Experimental Physics, Slovak Academy of Sciences, Košice, Slovak Republic ${ }^{f, j}$

18 School of Physics and Chemistry, University of Lancaster, Lancaster, $U K^{b}$

${ }^{19}$ Department of Physics, University of Liverpool, Liverpool, $U K^{b}$

${ }^{20}$ Queen Mary and Westfield College, London, $U K^{b}$

${ }^{21}$ Physics Department, University of Lund, Lund, Sweden ${ }^{g}$

${ }^{22}$ Department of Physics and Astronomy, University of Manchester, Manchester, $U K^{b}$

${ }^{23}$ CPPM, Université d'Aix-Marseille II, IN2P3-CNRS, Marseille, France

${ }^{24}$ Institute for Theoretical and Experimental Physics, Moscow, Russia

${ }^{25}$ Lebedev Physical Institute, Moscow, Russia ${ }^{f, k}$

${ }^{26}$ Max-Planck-Institut für Physik, München, Germany ${ }^{a}$

${ }^{27}$ LAL, Université de Paris-Sud, IN2P3-CNRS, Orsay, France

${ }^{28}$ LPNHE, École Polytechnique, IN2P3-CNRS, Palaiseau, France

${ }^{29}$ LPNHE, Universités Paris VI and VII, IN2P3-CNRS, Paris, France

${ }^{30}$ Institute of Physics, Academy of Sciences of the Czech Republic, Praha, Czech Republic ${ }^{f, h}$

${ }^{31}$ Nuclear Center, Charles University, Praha, Czech Republic ${ }^{f, h}$

32 INFN Roma 1 and Dipartimento di Fisica, Università Roma 3, Roma, Italy 
${ }^{33}$ Paul Scherrer Institut, Villigen, Switzerland

${ }^{34}$ Fachbereich Physik, Bergische Universität Gesamthochschule Wuppertal, Wuppertal, Germany $^{a}$

${ }^{35}$ DESY, Institut für Hochenergiephysik, Zeuthen, Germany ${ }^{a}$

${ }^{36}$ Institut für Teilchenphysik, ETH, Zürich, Switzerland ${ }^{i}$

${ }^{37}$ Physik-Institut der Universität Zürich, Zürich, Switzerland ${ }^{i}$

${ }^{38}$ Institut für Physik, Humboldt-Universität, Berlin, Germany ${ }^{a}$

${ }^{39}$ Rechenzentrum, Bergische Universität Gesamthochschule Wuppertal, Wuppertal, Germany ${ }^{a}$

${ }^{40}$ Vistor from Yerevan Physics Institute, Armenia

${ }^{41}$ Foundation for Polish Science fellow

${ }^{42}$ Dept. Fis. Ap. CINVESTAV, Mérida, Yucatán, México

${ }^{43}$ Institut für Experimentelle Kernphysik, Universität Karlsruhe, Karlsruhe, Germany

${ }^{a}$ Supported by the Bundesministerium für Bildung, Wissenschaft, Forschung und Technologie, FRG, under contract numbers 7AC17P, 7AC47P, 7DO55P, 7HH17I, 7HH27P, 7HD17P, 7HD27P, 7KI17I, 6MP17I and 7WT87P

${ }^{b}$ Supported by the UK Particle Physics and Astronomy Research Council, and formerly by the $U K$ Science and Engineering Research Council

${ }^{c}$ Supported by FNRS-FWO, IISN-IIKW

${ }^{d}$ Partially supported by the Polish State Committee for Scientific Research, grant no. 115/E-343/SPUB/P03/002/97 and grant no. 2P03B 05513

${ }^{e}$ Supported in part by US DOE grant DE F603 91 ER40674

${ }^{f}$ Supported by the Deutsche Forschungsgemeinschaft

${ }^{g}$ Supported by the Swedish Natural Science Research Council

${ }^{h}$ Supported by GA $\check{C} R$ grant no. 202/96/0214, GA AV $\check{C} R$ grant no. A1010821 and GA UK grant no. 177

${ }^{i}$ Supported by the Swiss National Science Foundation

${ }^{j}$ Supported by VEGA SR grant no. 2/5167/98

${ }^{k}$ Supported by Russian Foundation for Basic Research grant no. 96-02-00019 


\section{Introduction}

The most precise determinations of the gluon momentum distribution in the proton have been obtained so far from the analysis of scaling violations of the proton structure function $F_{2}$ [ [1], 2] . This method is however indirect in the sense that $F_{2}$ at low values of the Bjorken scaling variable $x_{B}$ actually probes the sea quark distributions which are related via the QCD evolution equations to the gluon distribution. The local behaviour of the structure function $F_{2}$ at a given value of $x_{B}$ depends on the gluon distribution $x_{g} g\left(x_{g}\right)$ in a rather wide range of values of the momentum fraction $x_{g}$, and the analysis requires the assumption of a certain functional form of $x_{g} g\left(x_{g}\right)$, the parameters of which are then determined in a fit procedure.

More direct determinations of the gluon density can be obtained by reconstruction of the kinematics of the interacting partons from the measurement of the hadronic final state in gluoninduced processes. Such direct measurements are complementary to the indirect analyses: although still limited in statistics, they are in principle more sensitive to local variations of the gluon distribution. They are subject to different systematic effects and provide an independent test of perturbative QCD. Direct gluon density determinations have previously been performed using events with $J / \Psi$ mesons in the final state [3] and dijet events [ 4 ].

The production of heavy quarks in electron-proton interactions proceeds, in QCD, almost exclusively via photon-gluon fusion, where a photon coupling to the incoming electron interacts with a gluon in the proton by forming a quark-anti-quark pair. This holds both for deep inelastic scattering (DIS) and for photoproduction where the exchanged photon is almost real. Differential charm photoproduction cross sections in the range of experimental acceptance were found to be reasonably well reproduced by such a description in Next to Leading Order (NLO) QCD [5], and measurements of the charm contribution to the deep inelastic proton structure function, $F_{2}^{c}$ [6], also confirm this picture.

Compared to the dijet case, smaller invariant masses of the partonic sub-process can be accessed in charm production. It is thus possible to extend the gluon density determination towards smaller momentum fractions $x_{g}$. The charm data are statistically less powerful, but there is also much less background from quark-induced processes.

Recently, Next-to-Leading-Order (NLO) QCD calculations of differential charm cross sections at HERA have become available, both for DIS [7, 8], and for the photoproduction regime [9]. With these calculations it becomes possible to determine $x_{g} g\left(x_{g}\right)$ directly. Here, measurements of differential $D^{*}$ production cross sections with the $\mathrm{H} 1$ detector are presented in both regimes, and from these results the gluon density is determined in NLO.

The theoretical framework and the reconstruction of the gluon momentum fraction will be described in more detail in the next section. In Section 3 the measurement of differential $D^{*}$ production cross sections will be presented separately for DIS and photoproduction. In each regime, the results will be compared with predictions based on the appropriate NLO QCD calculations. Section 4 will explain the method used to extract the gluon density from the measured cross sections, and will present the results. The paper concludes with a comparison to results obtained by $\mathrm{H} 1$ from the analysis of the structure function $F_{2}$. 


\section{Principle of the Analysis}

\subsection{NLO QCD calculations}

Differential charm production cross sections in $e p$ interactions have been calculated [10, 11] in NLO QCD in the $\overline{M S}$ renormalization scheme. The computations were carried out using the subtraction method, which is based on the replacement of divergent soft and collinear terms in the squared matrix elements by generalized distributions. Charm quarks are treated as massive particles appearing only in the final state whereas the three light flavours (and gluons) are the only active partons in the initial state ("massive" scheme, "Three Flavour Number" scheme). In Leading Order, the production proceeds via the partonic sub-process $\gamma g \rightarrow c \bar{c}$. In NLO, there are in addition contributions to the cross section associated with diagrams where a real gluon is radiated from a gluon or a quark line, $\gamma g \rightarrow c \bar{c} g$, there are quark induced contributions via the processes $\gamma q \rightarrow c \bar{c} q$ and $\gamma \bar{q} \rightarrow c \bar{c} \bar{q}$, and there are virtual corrections to the cross section.

For the photoproduction processes, resolved photon-proton interactions have to be taken into account in addition to the direct photon-proton scattering. While in the latter processes, the photon couples directly to a parton of the proton, the resolved photon processes involve the partonic structure of the photon itself. In NLO, the distinction of these processes is not unambiguous; here, a part of the photon parton density is contained in the direct photon calculation. The remaining part that depends on the parton density functions of the photon is termed resolved. In the calculations of charm production performed in the 'massive' scheme the direct contribution is dominant in the phase space where at least one charmed meson is produced in the detector acceptance. Previous measurements of charm photoproduction [5] have confirmed this.

Depending on the kinematic regime under consideration, the QCD scales in the theoretical calculations are governed by different quantities. In the DIS case the factorization scale is set to be $\mu_{F}=\sqrt{4 m_{c}^{2}+Q^{2}}$, where $m_{c}$ denotes the charm quark mass and $Q^{2}$ the virtuality of the exchanged photon, and the renormalization scale is set to be $\mu_{R}=\mu_{F}$. In the calculation of $\gamma p$ cross sections, the factorization scale is given by the transverse momenta of the charm quarks: $\mu_{F}=\sqrt{4 m_{c}^{2}+4 p_{\perp}^{2}}$, and the renormalization scale is chosen to be $\mu_{R}=\mu_{F} / 2$. Variation of these choices will be one means of estimating the uncertainties of the perturbative calculations.

Since the computations are differential in all relevant quantities, they provide access to the four-momenta of the outgoing partons. The evaluation of cross sections can be performed using Monte Carlo integrators. The calculations by Harris and Smith for DIS have been implemented in the HVQDIS program [8], and those for the photoproduction case by Frixione et al. in the FMNR package [9].

Charm quark hadronization into $D^{*}$ mesons is performed in the programs by convoluting the charm quark cross section with the Peterson fragmentation function [12]; the $D^{*}$ threemomentum vector is formed by scaling the quark three-momentum vector, and the $D^{*}$ energy is fixed such that the mass is $m\left(D^{*}\right)=2.01 \mathrm{GeV}$. Evolution of the fragmentation function, which one expects to become important for transverse $c$ quark momenta $p_{\perp} \gg m_{c}$, is not included because with present experimental cuts the region of interest is $p_{\perp} \sim m_{c}$. Cross sections for charm photoproduction at large transverse momenta $p_{\perp} \gg m_{c}$ have been computed 
in the"massless" scheme [13, 14], where charm acts as an active flavour in the initial state proton and photon.

Using the HVQDIS [8] and FMNR [9] programs, cross sections incorporating experimental cuts can be calculated in NLO as a function of any final state variable depending on the $D^{*}$ and electron four-momenta. Thus the predictions of the NLO QCD calculations can be confronted directly with measured differential cross-sections for inclusive $D^{*}$ production in the experimentally accessible kinematic range.

\subsection{Reconstruction of the gluon momentum fraction}

The kinematics of the Leading Order $2 \rightarrow 2$ process $\gamma g \rightarrow c \bar{c}$ is completely determined if the momenta of one incoming and one outgoing particle, here the photon and the charm (anti-) quark, are known. The momentum fraction $x_{g}$ in the infinite momentum frame is given by

$$
x_{g}=\frac{M^{2}+Q^{2}}{y \cdot s} \quad \text { with } \quad M^{2}=\frac{p_{\perp c}^{* 2}+m_{c}^{2}}{z(1-z)} \quad \text { and } \quad z \equiv \frac{p \cdot p_{c}}{p \cdot q}=\frac{\left(E-p_{z}\right)_{c}^{(\mathrm{Lab})}}{2 y E_{e}} \text {, }
$$

where $p_{\perp c}^{*}$ is the transverse momentum of the charm quark with respect to the photon-proton axis in the photon-proton centre-of-mass frame. The Lorentz invariant $z$ can be evaluated in the laboratory frame from the charm quark's energy $E$ and its momentum component $p_{z}$. 1 The variables $p, q$, and $p_{c}$ denote the four-momenta of the incoming proton, exchanged photon, and outgoing (anti-)charm quark, respectively, so that the usual DIS variables read $Q^{2}=-q^{2}$ and $y=(2 p \cdot q) / s ; \sqrt{s}$ is the $e p$ centre of mass energy, and $E_{e}$ the incoming electron energy in HERA. In DIS, Eq. 11 can be written in the form $x_{g}=x_{B}\left(1+M^{2} / Q^{2}\right)$, which relates $x_{g}$ to the Bjorken scaling variable $x_{B}$.

In the presence of gluon radiation (or a gluon transverse momentum in the initial state), relation (1) holds only approximately. Also, in the experiment one measures not quarks but $D^{*}$ mesons that on average carry only a fraction of the energy of the primarily produced $c$ quark. However, one can construct a hadronic observable $x_{g}^{O B S}$ that is well correlated with the true $x_{g}$ by replacing in Eq. 1]

$$
p_{\perp c}^{*} \rightarrow 1.2 p_{\perp D^{*}}^{*}, \quad\left(E-p_{z}\right)_{c} \rightarrow\left(E-p_{z}\right)_{D^{*}} .
$$

The factor 1.2 is introduced for convenience in the unfolding procedure described in Sect. 4 ; it is chosen such that $x_{g}^{O B S} \approx x_{g}$ on average.

\subsection{Analysis procedure}

The analysis proceeds in two main steps. First, the $D^{*}$ cross section is measured in the region of experimental acceptance as a function of various kinematic variables and of $x_{g}^{O B S}$. The data are corrected only for detector effects. As there is no extrapolation into unmeasured regions of

\footnotetext{
${ }^{1} \mathrm{H} 1$ uses a right-handed coordinate system with the $z$ axis defined by the incident proton beam, and the $y$ axis pointing upward.
} 
phase space nor are there any parton-hadron correlations involved, this step can be performed using the Leading Order Monte Carlo programs which describe the final state reasonably well. The resulting cross sections are then confronted with the NLO QCD predictions.

In the second step the cross section as a function of $x_{g}^{O B S}$ is unfolded to the true variable $x_{g}$ and the gluon density $x_{g} g\left(x_{g}, \mu_{F}^{2}\right)$ is extracted. This step is carried out in NLO QCD in the framework of the Three Flavour Number Scheme. The programs HVQDIS for DIS and FMNR for photoproduction are used to calculate the cross sections in the region of acceptance as a function of $x_{g}^{O B S}$ as well as the correlations between the hadronic and partonic quantities, $x_{g}^{O B S}$ and $x_{g}$, taking into account NLO effects and fragmentation.

\section{Cross Section Measurement}

\subsection{Experimental conditions}

The data used here have been collected in the years 1994 to 1996 with the H1 detector at the HERA collider, where $27.5 \mathrm{GeV}$ positrons (henceforth generically termed "electrons") collided with $820 \mathrm{GeV}$ protons, at a centre of mass energy of $\sqrt{s}=300 \mathrm{GeV}$.

The H1 detector and its trigger capabilities have been described in detail elsewhere [15]. Charged particles are measured by two cylindrical jet drift chambers [16, 17], mounted concentrically around the beam-line inside a homogeneous magnetic field of 1.15 Tesla, yielding particle charge and momentum from the track curvature in the polar angular range of $20^{\circ}<\theta<160^{\circ}$, where $\theta$ is measured using the central jet and two $z$ drift chambers and is defined with respect to the incident proton beam direction. One double layer of cylindrical multi-wire proportional chambers (MWPC) [18] with pad readout for triggering purposes is positioned inside and another one in between the two jet chambers. The tracking detector is surrounded by a fine grained liquid argon calorimeter [19], consisting of an electromagnetic section with lead absorbers and a hadronic section with steel absorbers. It covers polar angles between $4^{\circ}$ and $154^{\circ}$. In the backward region $\left(153^{\circ}<\theta<177.8^{\circ}\right)$ it is complemented by a lead scintillator "Spaghetti" calorimeter (SpaCal) [20] which is optimized for the detection of the scattered electron in the DIS kinematic range under consideration here and provides Time-ofFlight functionality for trigger purposes. Installed in 1995, it consists of an electromagnetic and a more coarsely segmented hadronic section. A four-layer drift chamber (BDC) [21] mounted on its front improves the angular measurement of the scattered electron.

The luminosity is determined from the rate of Bethe-Heitler ep $\rightarrow e p \gamma$ bremsstrahlung events. The luminosity system consists of an electron detector and a photon detector, located $33 \mathrm{~m}$ and $103 \mathrm{~m}$ from the interaction point in the electron beam direction, respectively. The system is also used to tag photoproduction events by detecting electrons scattered at small angles. In 1995 and 1996 a second electron tagging detector of the same type was added at $44 \mathrm{~m}$. It covers a much lower $y$-range. Therefore the two electron detectors cover different regimes of photon-proton centre-of-mass energies, $W_{\gamma p}$, and thus are complementary. Timeof-flight (TOF) systems in the forward and backward directions are used to reject beam gas background. 


\subsection{Extraction of the $D^{*}$ signal}

The reconstruction of $D^{*}$ mesons in the DIS and photoproduction regime follows closely the method described in the previously published measurement of $D^{*}$ production [5]. It makes use of the $D^{*}$ tagging technique [22], i.e. of the tight kinematical conditions in the decay chain $D^{*+} \rightarrow D^{0} \pi_{\text {slow }}^{+} \rightarrow\left(K^{-} \pi^{+}\right) \pi_{\text {slou }}^{+}$] for which the overall branching fraction is $2.62 \%$ [23]. Particle tracks passing some quality cuts and fulfilling transverse momentum cuts depending on the kinematic range, are first combined in unlike-sign charged pairs. No particle identification is applied. Assuming one track to be a kaon and the other to be a pion, the invariant mass is calculated for all combinations and each possible mass hypothesis assignment. Those $K^{-} \pi^{+}$pairs with an invariant mass consistent with the $D^{0}$ mass within $\pm 80 \mathrm{MeV}$ are combined with a third track (" $\pi_{\text {slow }}^{+}$") which has to have charge of opposite sign to that of the kaon candidate and to which is assigned the pion mass hypothesis. $D^{*+}$ production is found as a distinct enhancement in the distributions of the mass difference $\Delta M=M\left(K^{-} \pi^{+} \pi_{\text {slow }}^{+}\right)-M\left(K^{-} \pi^{+}\right)$around the expected mass difference of $145.4 \mathrm{MeV}$ [23]. The $\Delta M$ distributions for all $D^{*}$ candidates are shown in Fig. 1 for the DIS (a) and the two photoproduction samples $(b, c)$. No enhancement is observed if the mass difference for the wrong charge combinations $M\left(K^{-} \pi^{-} \pi^{+}\right)-M\left(K^{-} \pi^{-}\right)$ is used instead.

The number of events, $N_{D^{*}}$, is extracted from maximum likelihood fits to the distributions of the mass difference with a function taken to be a superposition of a Gaussian for the signal and a term $N_{B}\left(\Delta M-m_{\pi}\right)^{a}$ for the background. The position and width of the Gaussian are fixed to the values found from the total sample including both photoproduction and DIS events. The position is consistent with the expected value, and the width agrees with simulation results. The normalization of the signal, the background normalization $N_{B}$, and the shape exponent $a$ are free parameters in the fit. Uncertainties from the parameterization of signal and background are accounted for in the systematic error. From Monte Carlo simulations the contribution to the signal due to $D^{0}$ decays into channels other than $K^{-} \pi^{+}$(mostly $D^{0} \rightarrow K^{-} K^{+}$and $D^{0} \rightarrow \pi^{-} \pi^{+}$) is obtained to be $r=(3.5 \pm 1.5) \%$. For these reflections a correction is made.

\subsection{Acceptance and efficiency determination}

A Monte Carlo simulation is used to determine the detector acceptance and the efficiency of the reconstruction and the selection cuts. Electroproduction events and direct and resolved photoproduction events were generated in Leading Order with the AROMA 2.2 [24] and PYTHIA 5.7 programs [25]. The hadronization step was performed according to the Lund string model. The generated events were then processed by the $\mathrm{H} 1$ detector simulation program, and were subjected to the same reconstruction and analysis chain as the real data. The observed rapidity and transverse momentum distributions of $D^{*}$ mesons are acceptably reproduced. The changes found when varying the shape of the charm fragmentation function by using a Peterson function with $\epsilon_{c}$ ranging from 0.026 to 0.046 are accounted for in the systematic error. The dependence of the simulated efficiencies on other parameter choices made for the simulation (charm mass, parton density distributions) was studied, found to be small, and is neglected.

\footnotetext{
${ }^{2}$ The charge conjugate is always implied.
} 
Contributions from $b \bar{b}$ production, with subsequent decays of $b$ flavoured hadrons into $D^{*}$ mesons, have been calculated using the AROMA generator to be about $1.6 \%$ for DIS and even smaller for photoproduction. No subtraction is made; the quoted $D^{*}$ cross sections are inclusive. A systematic error accounts for the change of the efficiency due to a variation of the $b \bar{b}$ contribution within a factor of 5 of its predicted value, thus taking the preliminary result [26] into account, where an unexpectedly high $b \bar{b}$ cross section at HERA was found.

\subsection{Differential cross sections}

Differential cross sections as functions of kinematic variables are measured by dividing the data sample into bins and determining the numbers of $D^{*}$ mesons and the efficiencies in each bin using the $\Delta m$ fit and correction procedure as outlined above. The effects of limited detector resolution on the variables are small with respect to the chosen bin sizes and are corrected for using the Monte Carlo simulation.

The cross sections are determined in the ranges specified in Tab. 1, where $\eta=-\ln \tan (\theta / 2)$

Table 1: Kinematic ranges of the cross section measurements.

\begin{tabular}{|c||c|c|}
\hline DIS & $\gamma p$, ETAG44 & $\gamma p$, ETAG33 \\
\hline $2<Q^{2}<100 \mathrm{GeV}^{2}$ & $Q^{2}<0.009 \mathrm{GeV}^{2}$ & $Q^{2}<0.01 \mathrm{GeV}^{2}$ \\
$0.05<y<0.7$ & $0.02<y<0.32$ & $0.29<y<0.62$ \\
$p_{\perp}\left(D^{*}\right)>1.5 \mathrm{GeV}$ & $p_{\perp}\left(D^{*}\right)>2 \mathrm{GeV}$ & $p_{\perp}\left(D^{*}\right)>2.5 \mathrm{GeV}$ \\
$\left|\eta\left(D^{*}\right)\right|<1.5$ & $\left|\hat{y}\left(D^{*}\right)\right|<1.5$ & $\left|\hat{y}\left(D^{*}\right)\right|<1.5$ \\
\hline
\end{tabular}

denotes the pseudo-rapidity in the laboratory frame. In photoproduction, where the photonproton centre-of-mass frame moves along the $z$ axis, the rapidity in the laboratory frame, $\hat{y}=-\frac{1}{2} \ln \frac{E-p_{z}}{E+p_{z}}$, is conveniently used.

In the following, the DIS and photoproduction analyses are presented separately, since the scattered electron is measured in different detectors and since different methods are used to reconstruct the event kinematics. Different cuts in the $D^{*}$ selection reflect different combinatorial background levels in the kinematic regimes under consideration.

\subsubsection{Deep Inelastic Scattering analysis}

The data have been taken in the years 1995-96 after the installation of the SpaCal and the BDC. They correspond to a total integrated luminosity of $\mathcal{L}=9.7 \mathrm{pb}^{-1}$. The events have been triggered by an electromagnetic cluster in the SpaCal of at least $6.5 \mathrm{GeV}$ energy, in coincidence with a charged track signal from the MWPC and central drift chamber trigger.

The identification of electrons is similar to the procedure used in the inclusive structure function measurement [27]. Scattered electrons are identified as clusters in the SpaCal with energy $E_{e^{\prime}}>8 \mathrm{GeV}$, with a cluster radius $<3.5 \mathrm{~cm}$ consistent with electromagnetic energy 
deposition, and with a cluster centre matched by a charged track candidate in the BDC within $2.5 \mathrm{~cm}$. The scattering angle is required to be $\theta_{e^{\prime}}<177^{\circ}$.

The DIS kinematic variables are reconstructed using the "electron $(e)$ " and the " $e \Sigma$ " method [28]. In both methods

$$
Q^{2}=4 E_{e} E_{e^{\prime}} \cos ^{2} \frac{\theta_{e^{\prime}}}{2}
$$

whereas

$$
y_{e}=1-\left(E_{e^{\prime}} / E_{e}\right) \sin ^{2}\left(\theta_{e^{\prime}} / 2\right) \quad \text { and } \quad y_{e \Sigma}=\frac{2 E_{e} \sum_{h a d}\left(E-p_{z}\right)}{\left[\sum_{\text {all }}\left(E-p_{z}\right)\right]^{2}}
$$

where the sum in the denominator runs over all (liquid Argon and SpaCal) calorimetric signals as well as central drift chamber tracks, avoiding double counting. The sum in the numerator is over the same objects except those forming the scattered electron candidate. The results obtained with both methods are found to be in very good agreement. The $e \Sigma$ method needs smaller QED radiative corrections, and it is less sensitive to uncertainties in the electron energy. The absolute energy scale of the SpaCal was known to a precision of $\pm 2 \%$ (at $27 \mathrm{GeV}$ ) at the beginning of its operation in 1995, and to $\pm 1 \%$ in 1996. The $e \Sigma$ method is therefore used for the 1995 data. For the 1996 data, the $e$ method is used, which yields slightly improved average resolution in $x_{g}^{O B S}$.

For $D^{*}$ reconstruction, cuts of $p_{\perp}>300$ (120) $\mathrm{MeV}$ are imposed on the on the transverse momenta in the laboratory frame $K$ and $\pi\left(\pi_{\text {slow }}\right)$ candidates. In order to suppress combinatorial background, the range of $z$ (Eqs. 1.2) is restricted to $z>0.2$ for $p_{\perp}\left(D^{*}\right)<3 \mathrm{GeV}$. Variations found by applying or not applying this cut are accounted for in the systematic error.

A total of $N_{D^{*}}=583 \pm 35$ events is observed; a correction is made for a small fraction $r$ which is attributed to reflections (see Sect. 3.2). The efficiency for electron and $D^{*}$ reconstruction and selection is found to be $\epsilon_{\text {reco }}=42 \%$ on average, ranging from $34 \%$ in the lowest to $57 \%$ in the highest $x_{g}^{O B S}$ bin. The trigger efficiency is determined from data, by using independent trigger conditions. It is found to be $\epsilon_{\text {trig }}=98.6 \%$ on average for the 1995 running period, and $83.3 \%$ for 1996 when tighter track conditions were applied. The dependence of $\epsilon_{\text {trig }}$ on the event kinematics is small and is taken from simulations.

There is no indication of photoproduction background seen in the data; the electron energy and $y_{\Sigma}$ distributions are consistent with expectations for a pure sample of DIS events. From simulations of direct and resolved charm photoproduction, we expect less than 1 event within the cuts specified. No subtraction is therefore made and a $1 \%$ error attributed to this possible background source.

Radiative corrections have been calculated using the program HECTOR [29]. They amount to $\delta_{\text {rad }}=3 \%(7 \%)$ on average for the $e \Sigma(e)$ method and are below $10 \%$ in all bins. The cross section for the given range in $Q^{2}, y, p_{\perp}$ and $\eta$ (Tab. 1) is obtained as

$$
\sigma=\frac{N_{D^{*}}(1-r)}{\epsilon_{\text {trig }} \epsilon_{\text {rec }} B R\left(D^{*} \rightarrow K \pi \pi\right) \mathcal{L}\left(1+\delta_{\text {rad }}\right)}=(5.75 \pm 0.35 \pm 0.79) \mathrm{nb},
$$

where the first error is statistical, the second is systematic and is detailed in Tab. 2. One of 
Table 2: Systematic errors of the cross section measurements.

\begin{tabular}{|l|r|r|}
\hline & DIS & $\gamma p$ \\
\hline Trigger efficiency & $+4.3,-3.3 \%$ & $6.5 \%$ \\
\hline Track reconstruction & \multicolumn{2}{|c|}{$7.5 \%$} \\
\hline$D^{\star}$-Fit & $+3.7,-4.4 \%$ & $6 \%$ \\
\hline Reflections & \multicolumn{2}{|c|}{$1.5 \%$} \\
\hline$b$-production background & \multicolumn{2}{|c|}{$1 \%$} \\
\hline$\gamma p$ background & $+0,-1 \%$ & - \\
\hline Radiative corrections & $2.9 \%$ & - \\
\hline Energy scale elm. SpaCal & $3.3 \%$ & - \\
\hline Energy scale Had SpaCal $( \pm 7 \%)$ & $<1 \%$ & - \\
\hline Energy scale LAr $( \pm 4 \%)$ & $<1 \%$ & - \\
\hline Electron angle $( \pm 0.5 \mathrm{mrad})$ & $<1 \%$ & - \\
\hline BDC hit finding & $2 \%$ & - \\
\hline Electron tagger acceptance & - & $6 \%$ \\
\hline Luminosity & \multicolumn{2}{|c|}{$1.5 \%$} \\
\hline branching fraction & \multicolumn{2}{|c|}{$4 \%$} \\
\hline $\mathrm{Z}$ - cut & $+7.5,-6.6 \%$ & - \\
\hline Fragmentation & $+0.6 \%,-2.4 \%$ & $3 \%$ \\
\hline \hline Total & $+14.1,-13.4 \%$ & $15 \%$ \\
\hline \hline
\end{tabular}

the largest contributions in both analyses is due to the uncertainty in the track reconstruction efficiency. Other important sources of systematic errors include uncertainties in the trigger efficiency and in the $D^{*}$ signal extraction. In DIS, uncertainties related to the modelling of the $D^{*}$ distributions, as reflected in the dependence on the $z$ cut, are also sizable. All contributions show only slight variation with $x_{g}^{O B S}$.

In Fig. 目 the cross section results are presented as function of $p_{\perp}\left(D^{*}\right), p_{\perp}^{*}\left(D^{*}\right), \eta\left(D^{*}\right)$, and $Q^{2}$. Compared to the data are NLO QCD calculations performed with the HVQDIS program version 1.1 [8] using a charm mass $m_{c}=1.5 \mathrm{GeV}$. The parton density set CTEQ4F3 [30] is used, which is consistent with the Three Flavour Number Scheme as implemented in HVQDIS. The value for the QCD scale, $\Lambda^{(5)}=237 \mathrm{MeV}$, corresponds in the two-loop approximation the world average for the strong coupling constant, $\alpha_{s}\left(M_{\mathrm{Z}}\right)=0.119 \pm 0.002$ [23]. For the $c \rightarrow D^{*}$ fragmentation fraction, the value $27 \%$ is used, consistent with the average value of $(0.71 \pm 0.05) \%$ for the whole fragmentation and decay chain $c \rightarrow D^{*+} \rightarrow K^{-} \pi^{+} \pi^{+}$[31] and the value of $2.62 \%$ [23] used for the decay branching fraction. For the fragmentation parameter $\epsilon_{c}=0.036$ is used, which has been extracted [32] from ARGUS $e^{+} e^{-}$annihilation data using NLO QCD in a Fixed Order massive charm scheme.

The NLO QCD predictions (displayed as histograms) agree with the measurements except for $d \sigma / d \eta$ for which the calculations tend to undershoot the data at low $\eta$ and to overshoot at high $\eta$. This disagreement can be accommodated within acceptable ranges of parameters such as the QCD scale and parton density functions. The shaded band in the figures represents the uncertainty of the theoretical prediction arising from different choices of the charm mass between 1.3 and $1.7 \mathrm{GeV}$. 


\subsubsection{Photoproduction analysis}

Data are analyzed independently for the case where the scattered electron is detected in the electron tagger at $33 \mathrm{~m}$ and in the tagger at $44 \mathrm{~m}$. Henceforth, the respective data samples will be referred to as ETAG33 (recorded in 1994-96) and ETAG44 (1995-96). The total integrated luminosities of these samples are $10.7 \mathrm{pb}^{-1}$ for ETAG33 and $10.2 \mathrm{pb}^{-1}$ for ETAG44. The two samples have no events in common. The kinematic ranges within which the differential distributions are measured are given in Tab. 1.

The events were triggered by a coincidence of the $E T A G$ signals with track candidate signals obtained from the MWPC and central drift chamber trigger systems. Proton beam induced background is reduced by excluding events with energy flow only into the forward region of the detector.

The acceptances of the electron taggers and their trigger efficiencies are accounted for by assigning weights to individual events. The acceptance depends on $y$, which is reconstructed here using $y=1-E_{e^{\prime}} / E_{e}$. For the ETAG33 sample it is above $20 \%$ with an average value of about $60 \%$ in the specified range. This corresponds to a $\gamma p$ centre-of-mass energy range of $162 \mathrm{GeV}<W_{\gamma p}<234 \mathrm{GeV}$, with a mean of $\left\langle W_{\gamma p}\right\rangle=194 \mathrm{GeV}$ and an average $\left\langle Q^{2}\right\rangle \approx 10^{-3} \mathrm{GeV}^{2}$. The acceptance of the ETAG44 peaks sharply around $y=0.09$ and corresponds to an average of $\left\langle W_{\gamma p}\right\rangle=88 \mathrm{GeV}$. For ETAG44, an average value for the acceptance is used for all events.

The $K$ and $\pi$ candidates used in the $D^{*}$ reconstruction are required to have transverse momenta $p_{\perp}>0.5 \mathrm{GeV}$ (ETAG33) or $p_{\perp}>0.35 \mathrm{GeV}$ (ETAG44) respectively. A cut of $p_{\perp}>0.15 \mathrm{GeV}$ is imposed on the slow pion track. The method of equivalent event numbers [33] is used to determine the errors of the acceptance-weighted numbers of $D^{*}$ candidates. The weighted numbers of combinations found in the $\Delta m$ fits are $489 \pm 92$ (for ETAG33) and $299 \pm 75$ (for ETAG44).

Using the efficiencies for the track component of the trigger condition, as well as the $D^{*}$ reconstruction and selection efficiencies as determined by Monte Carlo simulations, the cross sections for the specified ranges are found using Eq. F. In the photoproduction case QED radiative corrections are negligible due to the requirement of no energy deposition in the photon detector of the luminosity system. Systematic errors (see Tab. 2) are similar to those of the DIS analysis, except for those related to the electron measurement. Here, uncertainties related to the electron tagger acceptance give a sizable contribution. The systematic errors are combined in quadrature to give $\pm 15 \%$.

Following the Weizsäcker-Williams Approximation (WWA) [34], the electroproduction cross section $\sigma_{e p}$ is converted into a photoproduction cross section according to $\sigma\left(\gamma p \rightarrow D^{*} X\right)=$ $\sigma(e p) / F$, where $F$ is the flux of photons emitted by the electron. For ETAG33 (ETAG44) the flux factor is $F=0.0128(0.0838)$.

The measured distributions $d \sigma\left(\gamma p \rightarrow D^{* \pm} X\right) / d \hat{y}$ are shown for ETAG33 and for ETAG44 in Fig. Ba and c, respectively, for the range specified in Tab. 1. The distributions $d \sigma_{\gamma p} / d p_{\perp}$ are shown in Fig. 3b,d. In addition, for ETAG33 the double-differential distribution $d^{2} \sigma_{\gamma p} / d \hat{y} d p_{\perp}$ is presented for three different ranges of transverse momentum in Fig. 4 . 
The superimposed histograms shown in Fig. 3 and in Fig. 4 represent the absolute predictions of the NLO QCD calculation [9], using, as in the DIS case, $m_{c}=1.5 \mathrm{GeV}, \epsilon_{c}=0.036$, and $27 \%$ for the $c \rightarrow D^{*}$ fragmentation fraction. The calculations are shown for the parton densities MRST1 [35] (proton) in combination with GRV-HO [36] (photon). The histograms for the ETAG33 sample are averages of calculations made at three representative $W_{\gamma p}$ values, weighted by the photon flux integrated over the represented range. For ETAG44 the calculation is performed at a fixed $W_{\gamma p}=88 \mathrm{GeV}$. Reasonable agreement is observed for both the shape and the absolute normalization between the measured single differential $p_{\perp}$ and $\hat{y}$ distributions and the NLO QCD calculation.

Fig. T reveals in more detail that the agreement is good in the low $p_{\perp}$ region where the "massive" QCD calculation is expected to describe most reliably the data, and where the bulk of the events is found. For comparison, also overlaid in Fig. 7 is a calculation performed in the "massless" scheme [14]. This approach is not expected to provide a description of the data in the region $p_{\perp} \sim m_{c}$. Fig. $\square$ shows that the shape of the measured rapidity distribution in the upper $p_{\perp}$ range of this analysis is less well described in both approaches.

\section{Determination of the Gluon Density}

The gluon density $x_{g} g\left(x_{g}\right)$ is extracted from the measured cross sections $d \sigma / d\left(\log x_{g}^{O B S}\right)$ which are compared to the NLO QCD predictions in Fig. 5.

The photoproduction data (Fig. 5b-d) are analyzed separately for three different photonproton energies $(88,185$, and $223 \mathrm{GeV})$. Here, the rapidity range is restricted to $-1.5 \leq \hat{y} \leq 0.5$ (for ETAG33) and $0 \leq \hat{y} \leq 1$ (for ETAG44). From the NLO calculation, the resolved photon contribution in this restricted range is expected to be less than 5\% (using the GRV-HO [36] or LAC1 [37] parton density set for the photon) in every bin and is accounted for in the systematic error.

The determinations of the gluon density in the proton from the DIS and from the photoproduction cross section follow the same principles. The calculation of the differential $D^{*}$ cross section in the experimentally accessible range $\sigma\left(x_{g}^{O B S}\right)$ can be written as

$$
\sigma\left(x_{g}^{O B S}\right)=\int d x_{g}\left[g\left(x_{g}, \mu^{2}\right) \cdot \hat{\sigma}\left(x_{g}, \mu^{2}\right) A\left(x_{g}^{O B S}, x_{g}, \mu^{2}\right)\right]+\sigma_{q u a r k}\left(x_{g}^{O B S}\right)
$$

where $\mu$ is the factorization scale, $\hat{\sigma}$ is the partonic cross section, and $A$ is the integration kernel which contains the effects of gluon radiation as well as fragmentation and also incorporates the limited kinematic range of the measured cross section. It receives the dominant contributions from regions where $x_{g} \approx x_{g}^{O B S}$. The quark-induced contribution to the cross section (in the range of acceptance) is denoted $\sigma_{\text {quark }}$. The form of Eq. 6 is such that a determination of the gluon density in the proton can be made from the measured cross section by means of an unfolding procedure.

Firstly, according to Eq. 6, the quark-induced contribution as predicted in the QCD calculation is subtracted from the data bin by bin. It is about $1 \%$ in total in DIS. The relative contribution rises with $x_{g}^{O B S}$, but remains below $10 \%$ in the highest DIS and below $20 \%$ in the 
highest $\gamma p$ bin. The correlation with the gluon density via QCD evolution can be neglected here, but the uncertainty arising from it is included in the error.

The quark-subtracted cross sections are converted into cross section distributions as a function of the true $x_{g}$ using the iterative unfolding procedure for binned distributions of Ref. [38]. The effects of limited detector resolution on $x_{g}^{O B S}$ are small with respect to the bin size and have already been corrected for in the measured cross section. The correlation between $x_{g}^{O B S}$ and $x_{g}$ as obtained from the QCD calculation is shown for the DIS case as a two-dimensional histogram in Fig. 6. This correlation is used for the first unfolding iteration and re-weighted for each subsequent iteration using the result of the previous one. After 4 steps at most, convergence is reached, and the "smeared" distribution $\sigma\left(x_{g}^{O B S}\right)$ agrees with the measurement.

The unfolded cross section distribution factorizes:

$$
\sigma\left(x_{g, i}\right) \sim g\left(x_{g, i}, \mu_{i}^{2}\right) \cdot \hat{\sigma}\left(x_{g, i}, \mu_{i}^{2}\right) ;
$$

but for every bin $i$ in $x_{g}$ (and $W$ ) the gluon density is probed at a different factorization scale $\mu_{i}$ which depends on the phase space region. The scale in the DIS measurement changes from $17 \mathrm{GeV}^{2}$ in the first to $28 \mathrm{GeV}^{2}$ in the last bin in $x_{g}$, the average being $\left\langle\mu^{2}\right\rangle=25 \mathrm{GeV}^{2}$. The $Q^{2}$ dependence of the cross section is well reproduced (see Fig. 22d). In photoproduction the factorization scale varies between 30 and $140 \mathrm{GeV}^{2}$, with an average value of $50 \mathrm{GeV}^{2}$, as can be seen in Fig. 7 where $g\left(x_{g}\right)$ as determined from the photoproduction data is plotted as a function of the scale $\mu$ associated to the $x_{g}$ and $W$ range of each measurement. To determine $g\left(x,\left\langle\mu^{2}\right\rangle\right)$ at an average scale, all data points are scaled to an average $\left\langle\mu^{2}\right\rangle$ :

$$
g\left(x_{g, i},\left\langle\mu^{2}\right\rangle\right)=\frac{g\left(x_{g, i},\left\langle\mu^{2}\right\rangle\right)^{\text {theory }}}{g\left(x_{g, i}, \mu_{i}^{2}\right)^{\text {theory }}} \cdot \frac{g\left(x_{g, i}, \mu_{i}^{2}\right)^{\text {theory }}}{\sigma\left(x_{g, i}\right)^{\text {theory }}} \cdot \sigma\left(x_{g, i}\right)^{\text {experiment }} .
$$

The change of $g\left(x_{g, i}, \mu^{2}\right)$ when varying the factorization scale from $\mu_{i}^{2}$ to $\left\langle\mu^{2}\right\rangle$ is taken from theory, assuming the same scale dependence as in CTEQ4 (DIS) or MRST1 $(\gamma p)$. This change is however small: in the DIS measurement $g\left(x, \mu_{i}^{2}\right)$ deviates from $g\left(x,\left\langle\mu^{2}\right\rangle\right)$ by no more than $9 \%$. In photoproduction, the variation can be inferred from Fig. 7. The effect is included in Eq. 8, and the dependence on the input parton density scale variation is accounted for in the systematic error.

The resulting gluon distribution, $x_{g} g\left(x_{g}\right)$, is shown in Fig. 8a for the DIS case at the average scale of $\mu^{2}=25 \mathrm{GeV}^{2}$. The covariance matrix for the statistical uncertainty has been calculated; the inner error bars in the figure represent the diagonal elements only. The correlation coefficients (defined as $\rho_{i j}=\operatorname{cov}_{i j} / \sqrt{\operatorname{cov}_{i i} \operatorname{cov}_{j j}}$ ) are 18\%, 27\%, 42\% for the first, second and third pair of neighbouring bins, respectively. An overall normalization uncertainty arises because of the experimental systematic error of the $D^{*}$ cross section. It is increased by the additional uncertainty from the $c \rightarrow D^{*}$ fragmentation fraction, and is added (in quadrature) to the statistical error to give the total experimental error shown as outer error bars. The downward extension of the error bar in Fig. \&a represents the decrease of $x_{g} g\left(x_{g}\right)$ if the $b \bar{b}$ contribution to the $D^{*}$ cross section is 5 times the size predicted by the AROMA Monte Carlo. The corresponding result from the photoproduction analysis is shown in Fig. $8 \mathrm{~b}$.

Theoretical uncertainties affecting the gluon density extraction are determined by re-calculating with the QCD programs for different parameter choices the cross sections and the correlations needed in the unfolding procedure. 
The dominant contribution in the DIS case arises from the uncertainty due to different choices of the charm quark mass between 1.3 and $1.7 \mathrm{GeV}$. In addition, the QCD factorization and renormalization scale $\mu$ was varied between $2 m_{c}$ and $2 \sqrt{4 m_{c}^{2}+Q^{2}}$ in DIS, following [8].

For the photoproduction results, the scale variation dominates the theoretical uncertainty over the whole range; a factor 2 is allowed in both directions. Here the charm quark mass uncertainty affects mainly the scale and is not added as a separate contribution.

One of the larger uncertainties is due to our ignorance of the charm fragmentation function which has not yet been measured in $e p$ scattering. It is assumed here that this can be taken from measurements in $e^{+} e^{-}$annihilation. A fragmentation parameter $\epsilon_{c}$ range between 0.026 and 0.046 is allowed in the calculation. Included in this range are the values extracted from ARGUS and OPAL data in a Fixed Flavour Number scheme [32]. The average uncertainty in $x_{g} g\left(x_{g}\right)$ due to this variation of the fragmentation parameter is $4 \%$ in DIS and $10 \%$ in photoproduction.

The uncertainty in the strong coupling constant (world average) is taken into account by variation of the scale $\Lambda^{(5)}$ between 213 and $263 \mathrm{MeV}$ [23]. In order to assess the dependence on the input parton density parameterization, the $\operatorname{MRS}\left(\mathrm{A}^{\prime}\right)$ [39] set is used. In photoproduction, an additional error of ${ }_{+0 \%}^{-4 \%}$ accounts for resolved contributions and the uncertainty in the photon structure function?.

The contributions to the theoretical error due to the discussed variations in the parameters of the NLO QCD calculations are added in quadrature and displayed as shaded histograms along the abscissa in Fig. 8. The errors at small $x_{g}$ are dominated by the statistical uncertainty.

The gluon densities extracted from DIS and photoproduction cross sections are in very good agreement with each other, as can be seen in Fig. 9. They can be compared with other results determined in NLO from other processes. For example, the result for $x_{g} g\left(x_{g}\right)$ determined by H1 [1] from a QCD analysis of the scaling violations of $F_{2}$ is also overlaid in Fig. 9. This result has been obtained from a fit to an inclusive measurement of the proton structure function $F_{2}$ using the NLO QCD evolution equations and the boson-gluon fusion prescription for the charm treatment. Within errors it agrees well with the gluon distributions determined in the analyses presented here, which are performed in the framework of the Three Flavour Number Scheme for DIS as implemented in the HVQDIS program, and in the "massive charm" calculations for photoproduction, implemented in the FMNR program. This amounts to an important verification of our understanding of the application of QCD in low $-x$ DIS and to a demonstration of the universality of the gluon distribution in the proton within the framework of such calculations at NLO.

\section{Conclusion}

Differential $D^{*}$ cross sections have been measured in deep inelastic ep scattering and in photoproduction. The results have been presented as a function of various kinematic variables and in different kinematic ranges. They have been compared to NLO QCD calculations in the "massive" Three Flavour Number Scheme. The calculations were performed using the programs

\footnotetext{
${ }^{3}$ In DIS, resolved contributions are expected to be negligible [40].
} 
HVQDIS and FMNR, which have implemented the Peterson form of the fragmentation function as determined from $e^{+} e^{-}$annihilation data to model the non-perturbative hadronization process. The overall description of the differential cross sections by the theoretical calculations has been found to be adequate.

Using the explicit NLO QCD calculations, the gluon density in the proton, $x_{g} g\left(x_{g}\right)$ has been determined from the measured cross sections as a function of $x_{g}^{O B S}$. The experimental observable $x_{g}^{O B S}$ is used as an estimator for the gluon momentum fraction $x_{g}$ using the kinematic information from the $D^{*}$ meson in addition to the scattered electron. The function $x_{g} g\left(x_{g}\right)$ has been extracted at an average scale $\mu^{2}=25 \mathrm{GeV}^{2}$. The gluon densities obtained from electroproduction and from photoproduction of charm agree well with each other and with that derived from the QCD analysis of the inclusive measurement of the proton structure function $F_{2}$.

\section{Acknowledgments}

We are grateful to the HERA machine group whose outstanding efforts have made and continue to make this experiment possible. We thank the engineers and technicians for their work in constructing and now maintaining the $\mathrm{H} 1$ detector, our funding agencies for financial support, the DESY technical staff for continual assistance, and the DESY directorate for the hospitality which they extend to the non-DESY members of the collaboration. We wish to thank S. Frixione, B. Kniehl, B. Harris and J. Smith for support in the QCD calculations, and P. Nason and C. Oleari for advice on fragmentation issues.

\section{References}

[1] S. Aid et al. (H1 Collaboration), Nucl. Phys. B470 (1996) 3.

[2] J.Breitweg et al. (ZEUS Collaboration), preprint DESY 98-121 (1998).

[3] D.Allasia et al. (NMC Collaboration), Phys. Lett. B258 (1991) 493.

[4] S. Aid et al. (H1 Collaboration), Nucl. Phys. B449 (1995) 3.

[5] S. Aid et al. (H1 Collaboration), Nucl. Phys. B472 (1996) 32.

[6] J. Aubert et al. (EMC Collaboration), Nucl. Phys. B213 (1983) 31;

C. Adloff et al. (H1 Collaboration), Z. Phys. C72 (1996) 593;

J. Breitweg et al. (ZEUS Collaboration), Phys. Lett. B 407 (1997) 402.

[7] B.W. Harris, hep-ph/9608379, talk presented at the 1996 annual meeting of the Division of Particles and Fields, American Physical Society, Minneapolis, USA, August 1996.

[8] B.W. Harris and J. Smith, Phys. Rev. D57 (1998) 2806.

[9] S. Frixione, M.L. Mangano, P. Nason, and G. Ridolfi, Phys. Lett. B308 (1993) 137;

S. Frixione, P. Nason, and G. Ridolfi, Nucl. Phys. B454 (1995) 3. 
[10] R.K. Ellis and P. Nason, Nucl. Phys. B312 (1989) 551;

J. Smith and W.L. Van Neerven, Nucl. Phys. B374 (1992) 36;

S. Frixione, M.L. Mangano, P. Nason, and G. Ridolfi, Nucl. Phys. B412 (1994) 225.

[11] B.W. Harris and J. Smith, Nucl. Phys. B452 (1995) 109; Phys. Lett. B353 (1995) 535.

[12] C. Peterson, D. Schlatter, I. Schmitt, and P.M. Zerwas, Phys. Rev. D 27 (1983) 105.

[13] B.A. Kniehl, G. Kramer, M. Spira, Z. Phys. C76(1997) 689;

M. Cacciari and M. Greco, Phys. Rev. D55 (1997) 7134;

J. Binnewies, B.A. Kniehl, G. Kramer, Z. Phys. C76(1997) 677.

[14] J. Binnewies, B.A. Kniehl, G. Kramer, Phys. Rev. D 58 (1998) 014014.

[15] I. Abt et al. (H1 Collaboration) Nucl. Instr. Meth. A386 (1997) 310 and 348.

[16] J. Bürger et al., Nucl. Instr. Meth. A279 (1989) 217.

[17] I. Abt et al. (H1 Coll.), Phys. Lett. B328 (1994) 176.

[18] K. Müller et al., Nucl. Instr. Meth. A312 (1992) 457.

[19] B. Andrieu et al., Nucl. Instr. Meth. A336 (1993) 460.

[20] R.D. Appuhn et al. (H1 SpaCal group), Nucl. Instrum. Meth. A386 (1997) 397.

[21] H1 Collaboration, Technical proposal for the upgrade of the backward region of the $\mathrm{H} 1$ detector, DESY internal report PRC-93/02.

[22] G. Feldmann et al., Phys. Rev. Lett. 38 (1977) 1313.

[23] C. Caso et al. (Particle Data Group), Eur. Phys J. C3 (1998) 1.

[24] G. Ingelman, J. Rathsman and G.A. Schuler, Comput. Phys. Commun. 101 (1997) 135.

[25] T. Sjöstrand, CERN-TH-6488 (1992), Comp. Phys. Comm. 82 (1994) 74.

[26] U. Langenegger (H1 Collaboration), talk given at "XXXIII Rencontres de Moriond: QCD”, Les Arcs, France, March 1998, to appear in the proceedings thereof;

H1 Collaboration, contributed paper no. 575 to the International Conference on High Energy Physics, Vancouver, Canada, July 1998.

[27] C. Adloff et al. (H1 Collaboration), Nucl. Phys. B497 (1997) 3.

[28] U. Bassler and G. Bernardi, Nucl. Instrum. Meth. A361 (1995) 197.

[29] A. Arbuzov, D. Bardin, J. Bluemlein, L. Kalinovskaya, and T. Riemann, Comp. Phys. Comm. 94 (1996) 128.

[30] H. Lai et al., Phys. Rev. D 55 (1997) 1280.

[31] ALEPH, DELPHI, L3 and OPAL Collab., Nucl. Instrum. Meth. A378 (1996) 101. 
[32] P. Nason and C. Oleari, preprint CERN-TH/98-339 (1998).

[33] G.Zech, preprint DESY 95-113 (1995).

[34] C.F. Weizsäcker, Z. Phys. 88 (1934) 612;

E.J. Williams, Phys. Rev. 45 (1934) 729.

[35] A.D. Martin, R.G. Roberts, W.J. Stirling and R.S. Thorne, Eur. Phys. J. C4 (1998) 463.

[36] M. Glück, E. Reya, and A. Vogt, Phys. Rev. D45 (1992) 3986, D46 (1992) 1973.

[37] A. Levy, H. Abramowicz and K. Charchula, Phys. Lett. B269 (1991) 458.

[38] G. D’Agostini, Nucl. Instrum. Meth. A362 (1995) 487.

[39] A.D. Martin, R.G. Roberts and W.J. Stirling, Phys. Lett. B354 (1995) 155.

[40] M. Glück, E. Reya, M. Stratman, Phys. Rev. D54 (1996) 5515. 

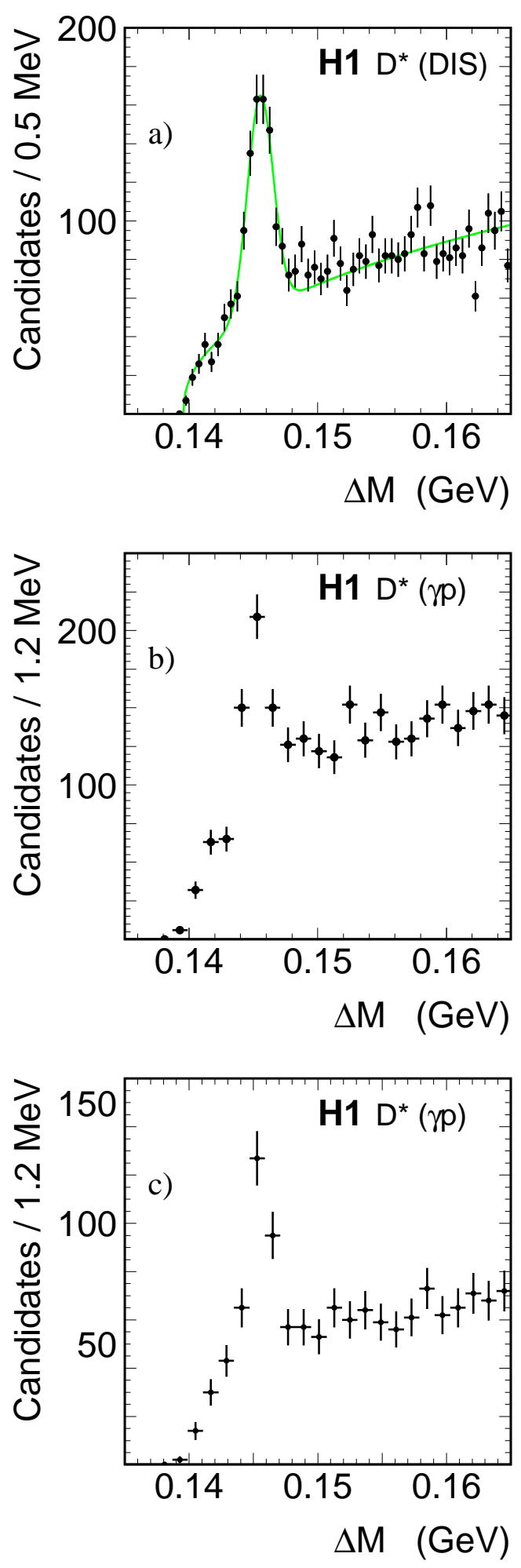

Figure 1: (a) Mass difference $\Delta M=M\left(K^{-} \pi^{+} \pi_{\text {slow }}^{+}\right)-M\left(K^{-} \pi^{+}\right)$distribution of $D^{*}$ candidates in DIS. The solid line represents the result of a fit as described in the text, which is used to extract the $D^{*}$ cross section. For the photoproduction samples ETAG33 (b) and ETAG44 (c) the unweighted $\Delta M$ distributions are shown. 

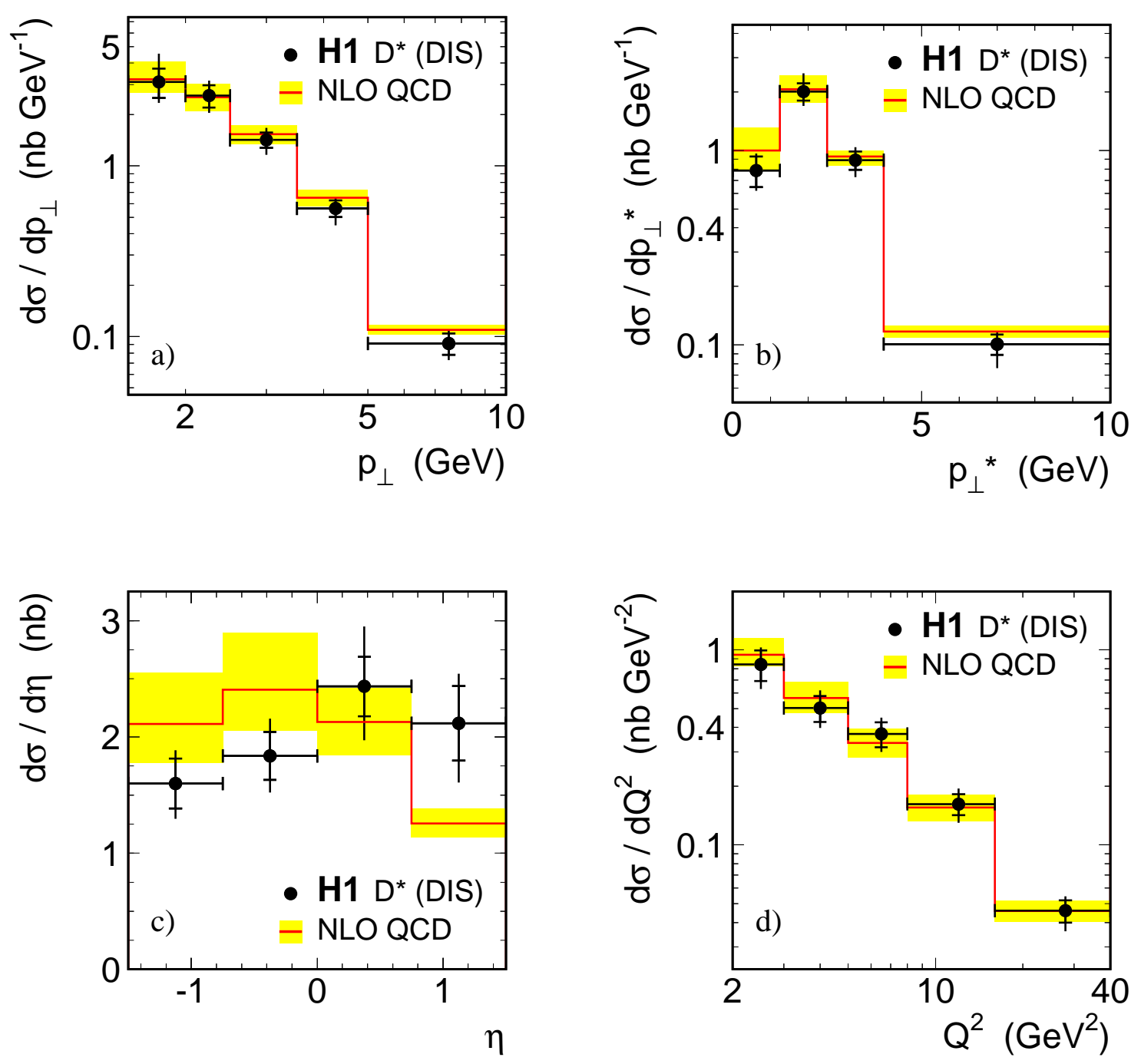

Figure 2: Differential DIS cross sections in the kinematic range of experimental acceptance (see Tab. [1). The H1 data are shown as points with error bars (inner: statistical, outer: total); the NLO QCD prediction using the CTEQ4F3 parton distribution set and a charm mass $m_{c}=1.5 \mathrm{GeV}$ is shown as a histogram. The shaded band represents the variation of the theoretical cross sections due to different choices of $m_{c}$ between 1.3 and $1.7 \mathrm{GeV}$. (a) Transverse $D^{*}$ momentum in the laboratory frame, (b) Transverse $D^{*}$ momentum in the hadronic centre-of-mass frame, (c) $D^{*}$ pseudo-rapidity, (d) four-momentum transfer squared, $Q^{2}$. 

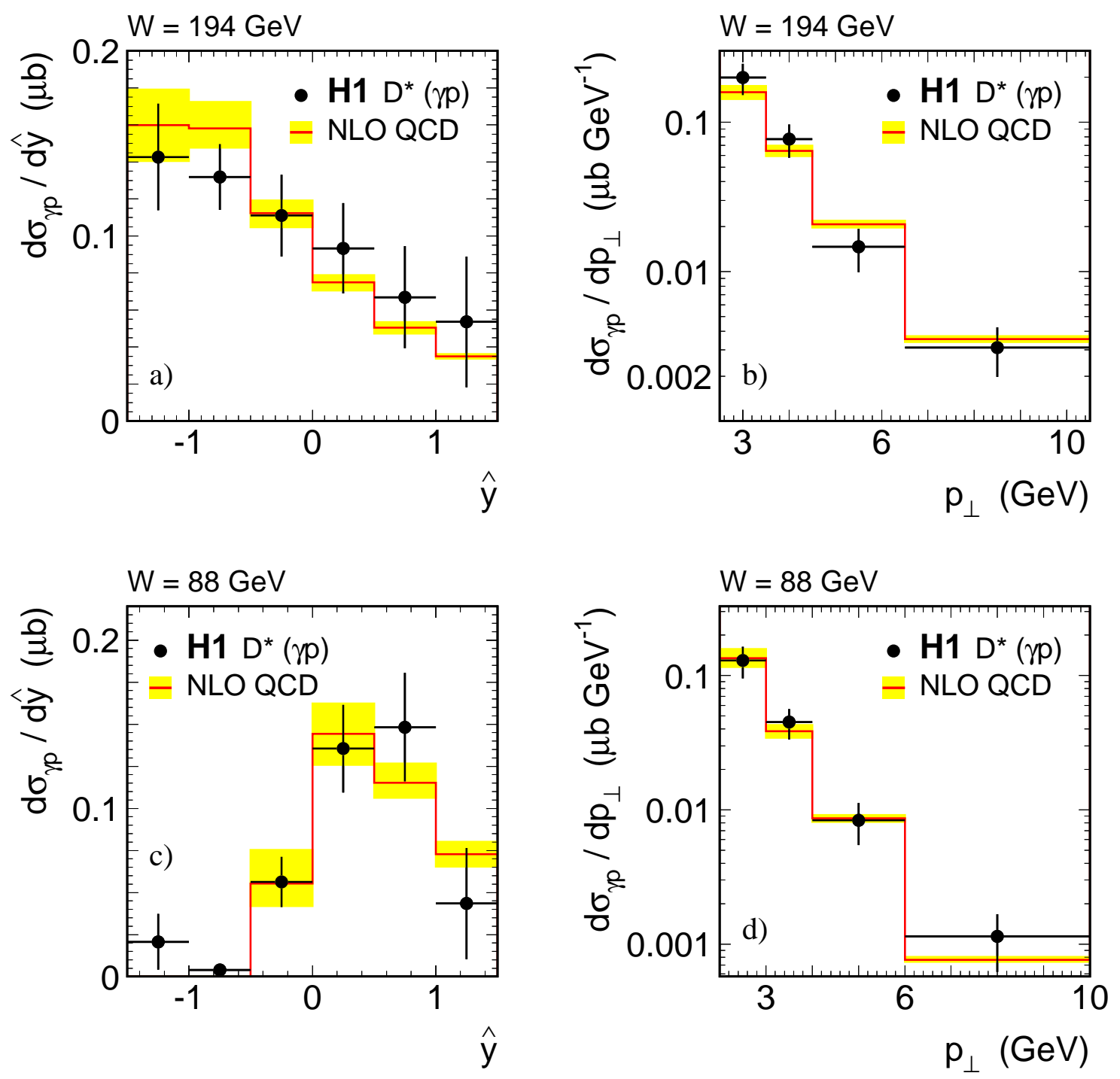

Figure 3: Differential photoproduction cross sections in the kinematic range of experimental acceptance (see Tab. 1). The data are shown as solid dots with statistical error bars for the ETAG33 sample $(a, b)$ and for the ETAG44 sample (c,d). A common systematic error of $15 \%$ is not shown. The histograms show the NLO QCD predictions based on the FMNR program, using the MRST1 parton density parameterization for the proton and the GRV-HO set for the photon. The width of the shaded band represents the variation of the theoretical cross sections due to different choices of $m_{c}$ between 1.3 and $1.7 \mathrm{GeV}$. 

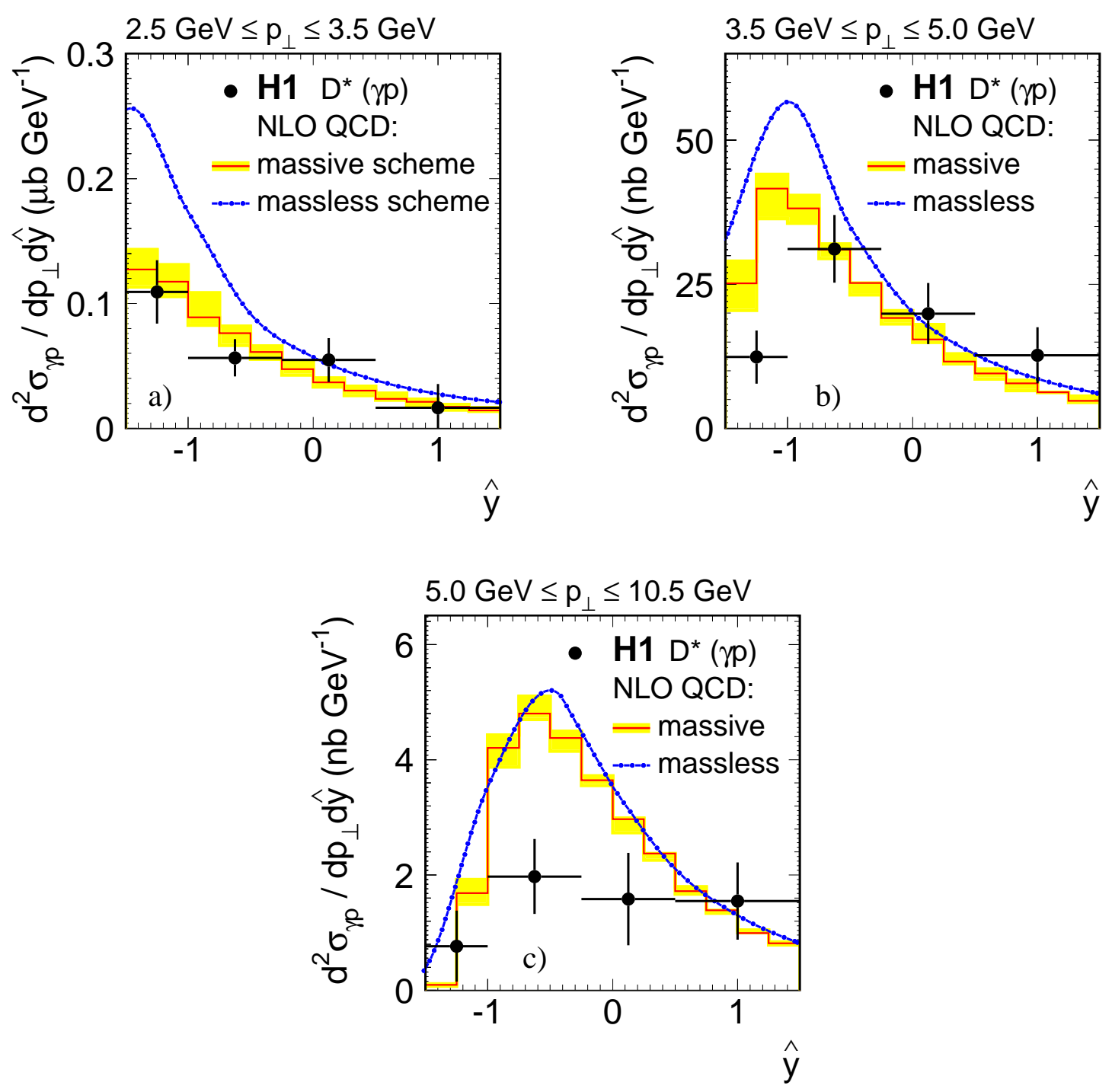

Figure 4: Double-differential cross section $d^{2} \sigma_{\gamma p} / d \hat{y} d p_{\perp}$ for three different ranges of transverse momentum at an average $W_{\gamma p}=194 \mathrm{GeV}$, shown as data points with statistical error bars. A common systematic error of $15 \%$ is not shown. The histograms represent the NLO QCD predictions in the massive scheme based on the FMNR program (using the parton density sets MRST1 (proton) and GRV-HO (photon)); the shaded bands show their variations due to different choices of the charm quark mass between 1.3 and $1.7 \mathrm{GeV}$. The curve shows the result of a NLO QCD calculation in the massless scheme using the CTEQ4M parton density set for the proton and GRV-HO for the photon, and a charm fragmentation function extracted from $e^{+} e^{-}$ in the same framework. 

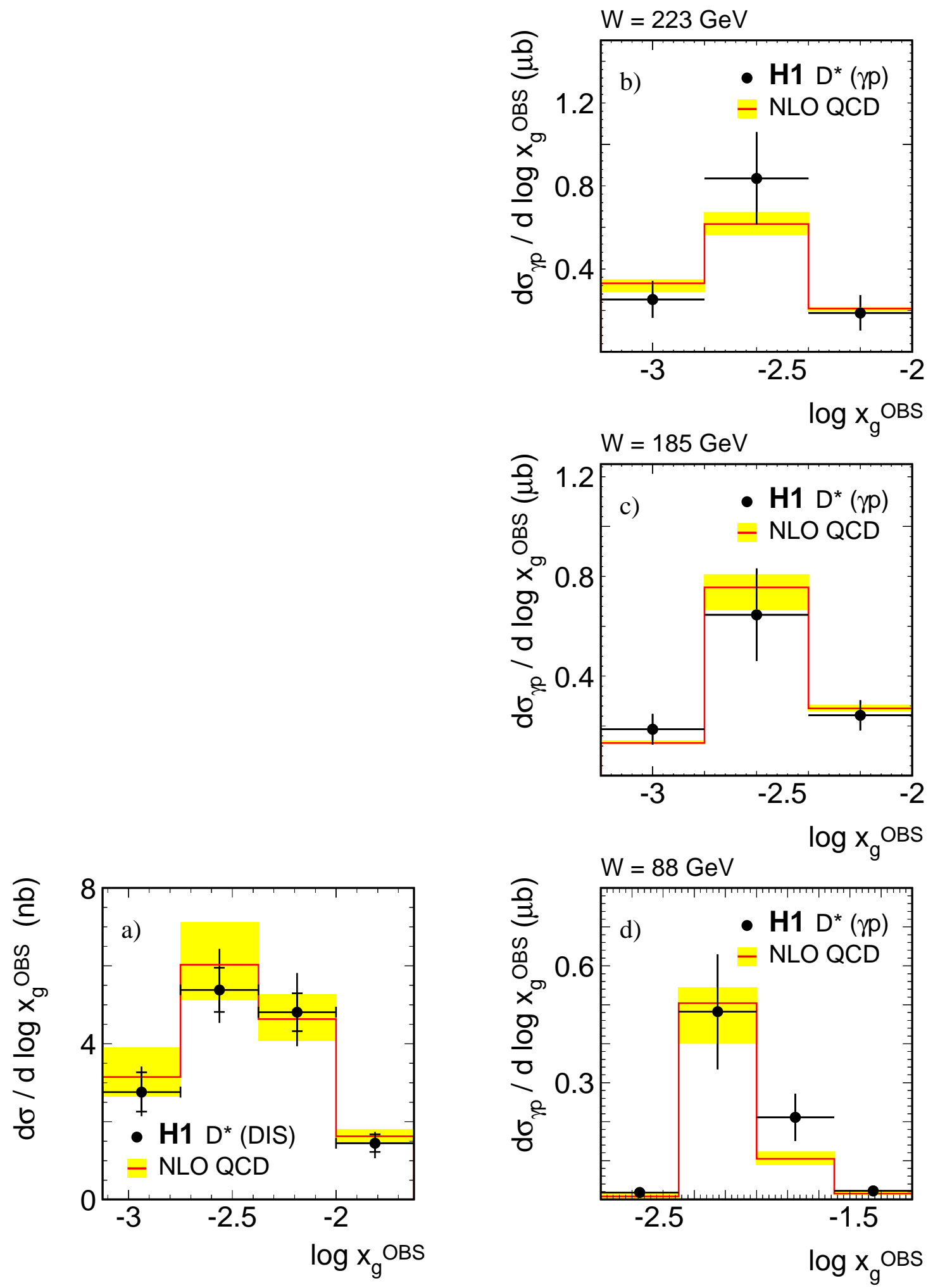

Figure 5: (a) Differential DIS cross section as a function of $x_{g}^{O B S}$ in the kinematic range of experimental acceptance (see Tab. 1). Figures (b-d) show the corresponding photoproduction cross sections, separately for $W_{\gamma P}=223,185$ and $88 \mathrm{GeV}$, respectively. The meaning of the data points, histograms and their errors is the same as in Fig. 2 (for DIS) and Fig. 3 (for $\gamma p$ ). 


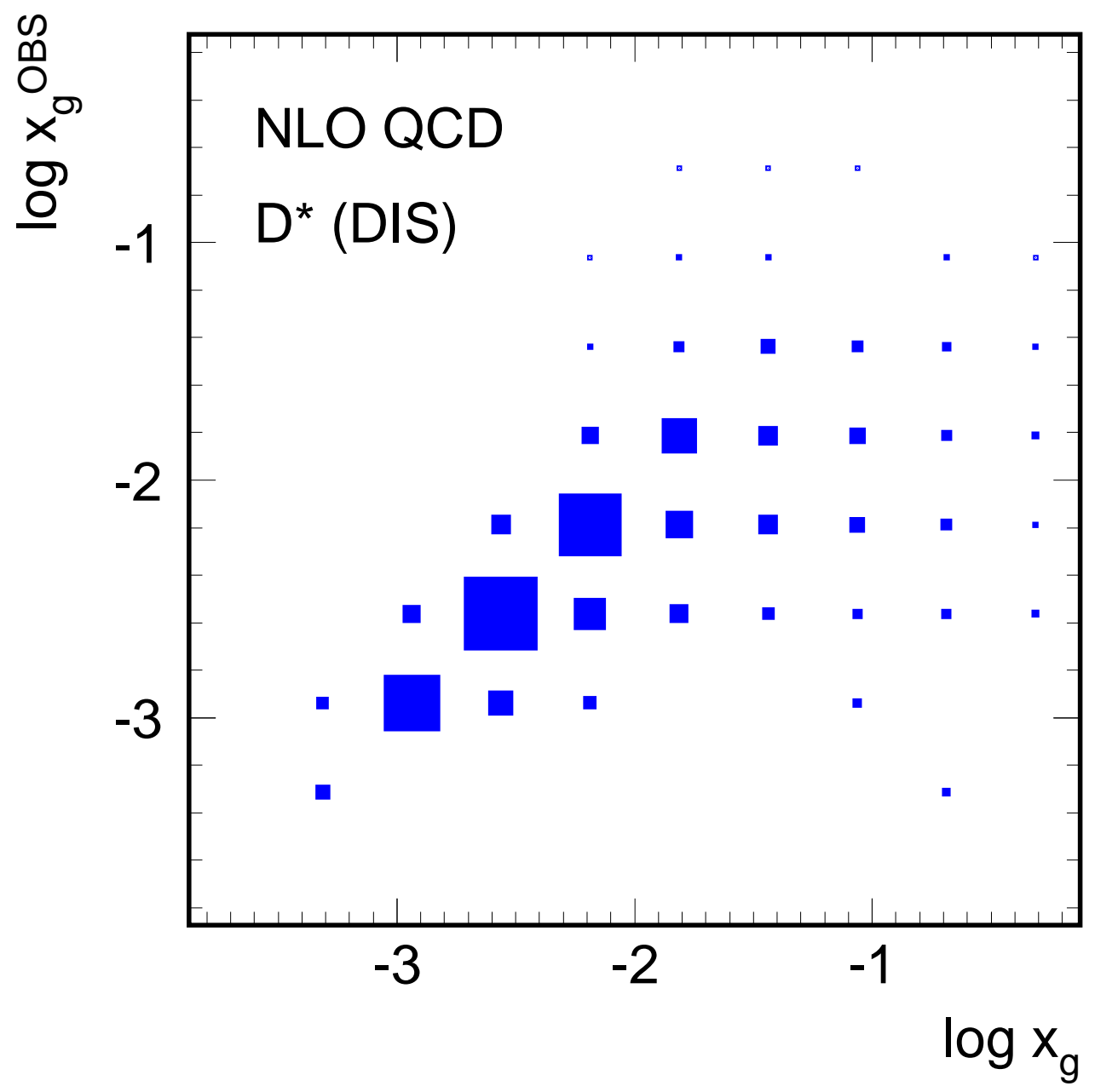

Figure 6: Correlation between the observable $x_{g}^{O B S}$ — calculated using Eqs. 1.2- and the true momentum fraction $x_{g}$, as predicted in NLOQCD. The bin area corresponds to the cross section contribution from the $x_{g}$ interval to the observed $x_{g}^{O B S}$ range. The correlation is distorted due to the effects of gluon radiation and fragmentation. 


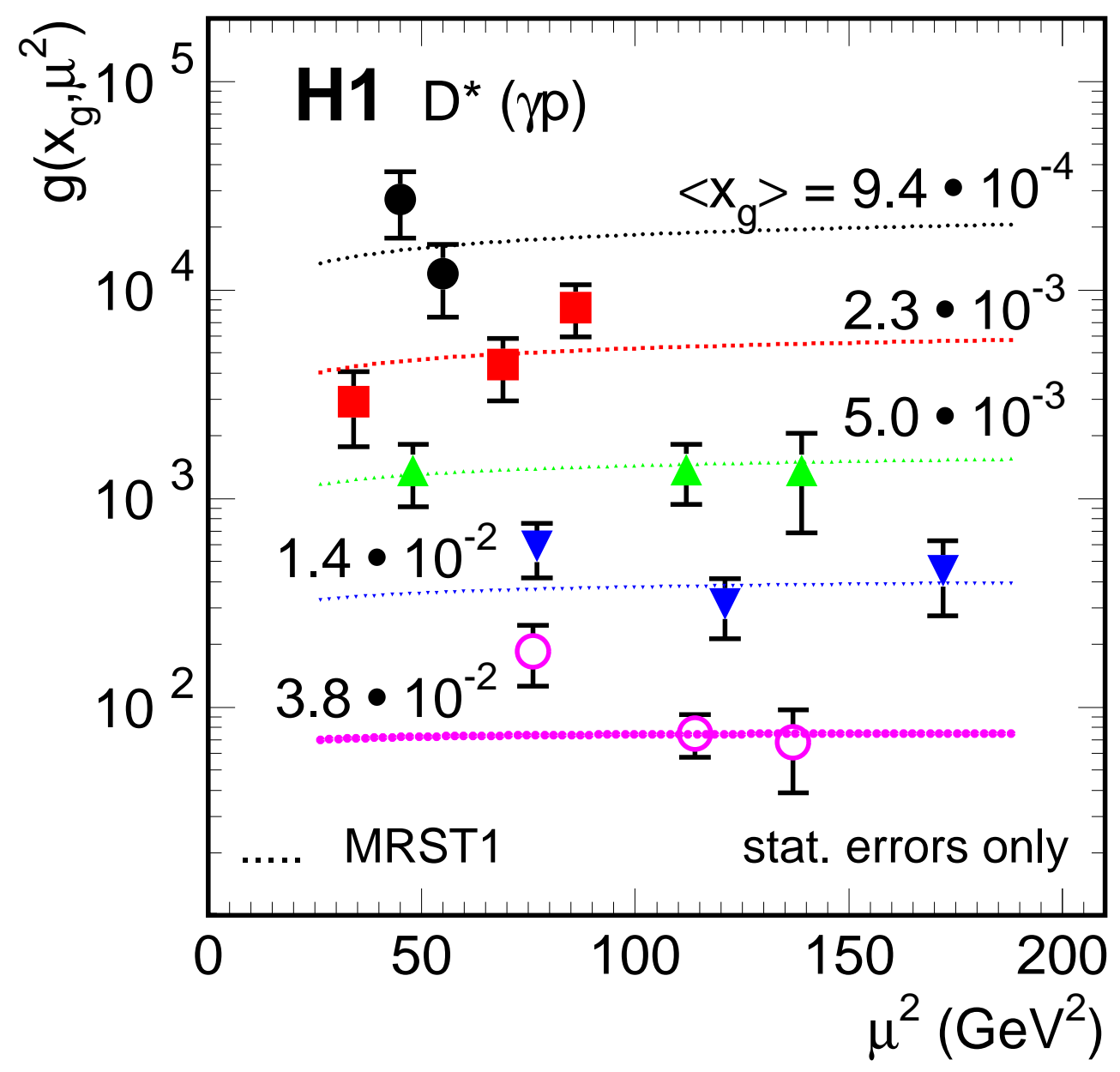

Figure 7: Gluon density distribution, measured in photoproduction as a function of the scale $\mu^{2}$. Data are plotted as $g\left(x_{g}, \mu^{2}\right)$ and not $x_{g} g\left(x_{g}, \mu^{2}\right)$, in order to show most clearly the evolution with scale. Each key symbol represents a different bin in $x_{g}$ with the average value given in the figure. The error bars represent the statistical errors only. The dotted lines represent the gluon density of the MRST1 parameterization, shown as a function of scale $\mu^{2}$ for the same set of $x_{g}$ values. 

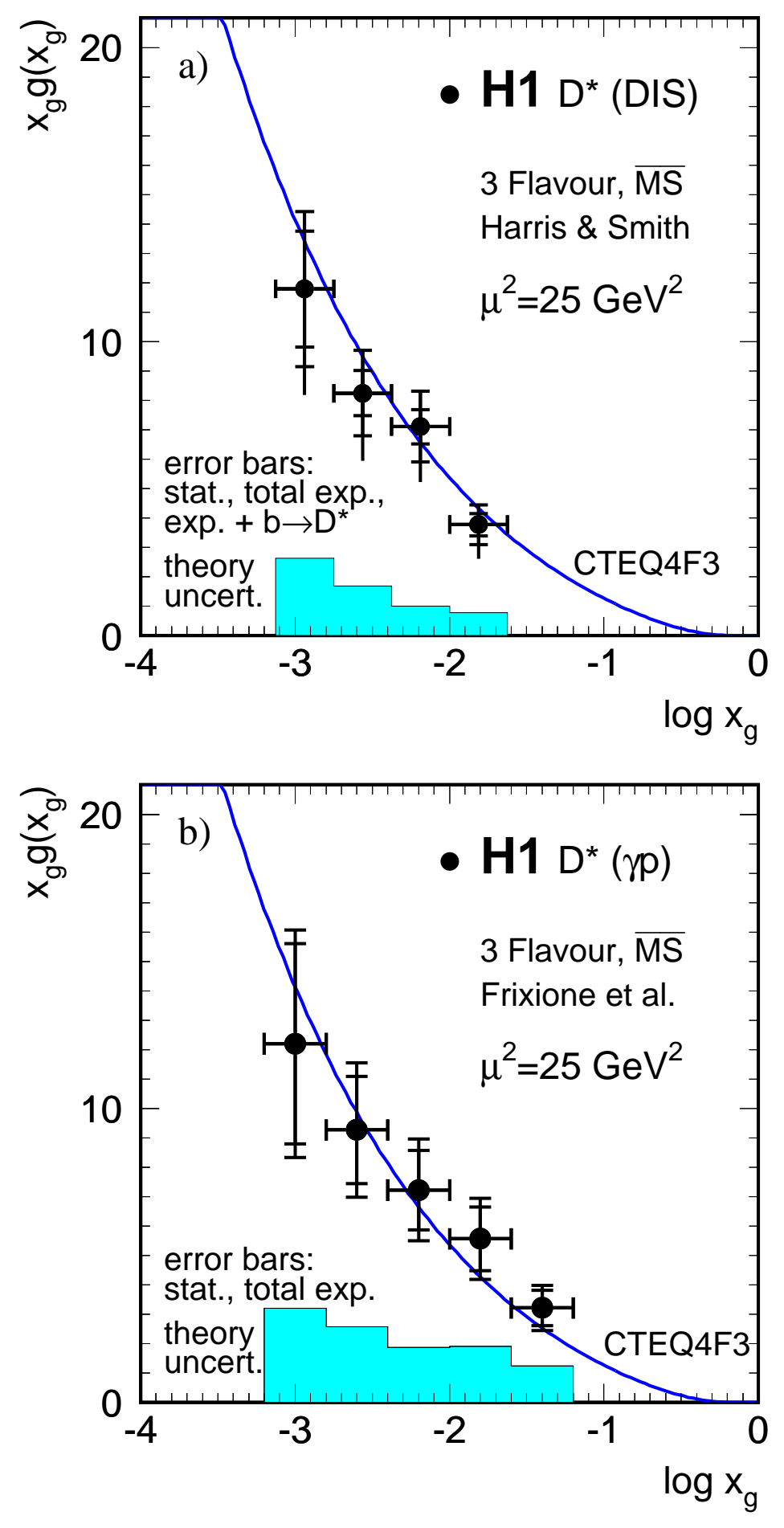

Figure 8: Gluon density, determined from DIS (a) and from from photoproduction data (b), compared to the CTEQ4F3 parameterization at $\mu^{2}=25 \mathrm{GeV}^{2}$ (full line). The error bars represent the statistical (inner) and total experimental (outer) error; in addition the effect of a subtraction of $b \bar{b}$ background (if 5 times higher than predicted in a LO Monte Carlo program) is displayed in (a), in (b) the $b \bar{b}$ contribution is negligible. The theoretical systematic error is shown as shaded band along the abscissa and is dominated in DIS (a) by the uncertainty associated with the charm quark mass, in photoproduction (b) it is dominated by the uncertainty associated with the renormalization and factorization scale. 


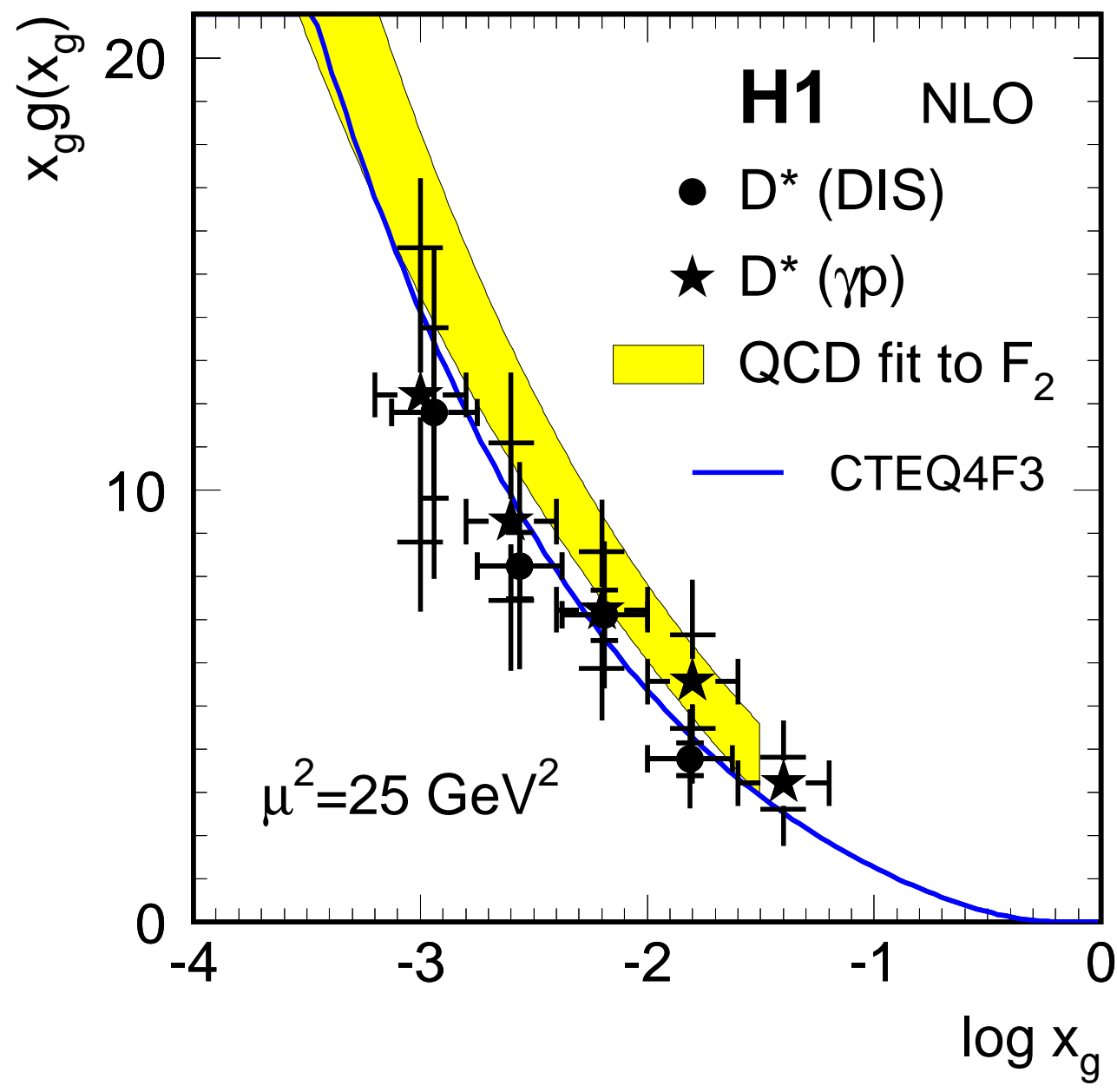

Figure 9: Gluon densities obtained from the two $D^{*}$ analyses. The inner error bars represent the statistical and the outer the total error. The systematic error is a quadratic sum of all contributions, dominated by the theoretical uncertainty. Both results are compared to the result of the H1 QCD analysis of the inclusive $F_{2}$ measurement [1] at $\mu^{2}=25 \mathrm{GeV}^{2}$ (light shaded band) and the CTEQ4F3 parameterization. 